\title{
Olfactory receptor and circuit evolution promote host specialisation
}

\author{
Thomas O. Auer ${ }^{1}{ }^{\star}$, Mohammed A. Khallaf ${ }^{2}$, Ana F. Silbering ${ }^{1}$, Giovanna Zappia ${ }^{1}$, Kaitlyn \\ Ellis $^{3}$, Raquel Álvarez-Ocaña ${ }^{1}$, J. Roman Arguello4, Bill S. Hansson ${ }^{2}$, Gregory S.X.E. \\ Jefferis $^{5}$, Sophie J.C. Caron ${ }^{3}$, Markus Knaden ${ }^{2}$, Richard Benton ${ }^{1, *}$
}

${ }^{1}$ Center for Integrative Genomics, Faculty of Biology and Medicine, University of Lausanne, $\mathrm{CH}-1015$, Lausanne, Switzerland ${ }^{2}$ Department of Evolutionary Neuroethology, Max Planck Institute for Chemical Ecology, D-07745, Jena, Germany ${ }^{3}$ Department of Biology, University of Utah, Salt Lake City, Utah, 84112, USA ${ }^{4}$ Department of Ecology and Evolution, Faculty of Biology and Medicine, University of Lausanne, $\mathrm{CH}-1015$, Lausanne, Switzerland ${ }^{5}$ Division of Neurobiology, MRC Laboratory of Molecular Biology, Cambridge, CB2 0QH, UK

\section{Summary}

\begin{abstract}
The evolution of animal behaviour is poorly understood ${ }^{1,2}$. Despite numerous correlations of behavioural and nervous system divergence, demonstration of the genetic basis of interspecific behavioural differences remains rare ${ }^{3-5}$. Here, we develop a novel neurogenetic model, Drosophila sechellia, a close cousin of $D$. melanogaster ${ }^{6,7}$ that displays profound behavioural changes linked to its extreme specialisation on noni fruit ${ }^{8-16}$. Using calcium imaging, we identify $D$. sechellia olfactory pathways detecting host volatiles. Mutational analysis indicates roles for different olfactory receptors in long- and short-range attraction to noni. Cross-species allele transfer demonstrates that tuning of one of these receptors is important for species-specific host-seeking. We identify the molecular determinants of this functional change, and characterise their evolutionary origin and behavioural significance. Through circuit tracing in the D. sechellia brain,
\end{abstract}

Users may view, print, copy, and download text and data-mine the content in such documents, for the purposes of academic research, subject always to the full Conditions of use:http://www.nature.com/authors/editorial_policies/license.html\#terms

*Corresponding authors: T: ++41 21692 3932, Richard.Benton@unil.ch, Thomas.Auer@unil.ch.

Data, code and biological material availability statement

All relevant data supporting the findings of this study are available from the corresponding authors upon request or, for behavioural experiments, included here as source data (Fig. 1b,d; Fig. 2c,d,e; Fig. 3d,e; ED Fig. 1c,e,f,g,h; ED Fig. 7e,f,g). Supplementary Table 7 lists the exact $\mathrm{n}$ and mean values for all electrophysiological data. Code used for analyses and all unique biological materials generated in this study (e.g., mutant and transgenic Drosophila strains) are available from the corresponding authors upon request.

\section{Author Contributions}

T.O.A. and R.B. conceived the project. All authors contributed to experimental design, analysis and interpretation of results. T.O.A. generated all molecular reagents and new drosophilid mutants and transgenic lines; other experimental contributions were as follows: T.O.A. (Fig. 1c; Fig. 2a,b,d,e; Fig. 3a-e; Fig. 4a-d,g; ED Fig. 1a-f; ED Fig. 2c; ED Fig. 3; ED Fig. 4c,e; ED Fig. 5; ED Fig. 6; ED Fig. 7a-f; ED Fig. 8; ED Fig. 9; ED Fig. 10a-d; ED Fig. 11f-m; ED Fig. 12), M.A.K. with input from B.S.H. and M.K. (Fig. 1b,d; Fig. 2c; Fig. 3d,f; ED Fig. 1f-h; ED Fig. 2a,b,d,e; ED Fig. 7g, Sup. Table 1), G.Z. (Fig. 1c; Fig. 2d; Fig. 4b; ED Fig. 1a,b; ED Fig. 3b; ED Fig. 5c,e,g; ED Fig. 6; ED Fig. 7a,b,f; ED Fig. 10b; ED Fig. 11k), A.F.S. (Fig. 1e,f; ED Fig. 4d,f,g), K.E. with input from S.J.C.C. (Fig. 4eg; ED Fig. 10e), R.A.O. (ED Fig. 4a,b), J.R.A (ED Fig. 11d,e), G.S.X.E.J. (Fig. 4c), R.B. (Fig. 4c). T.O.A. and R.B. wrote the paper with input from all other authors.

Competing interests

The authors declare no competing interests. 
we find that receptor adaptations are accompanied by increased sensory pooling onto interneurons and novel central projection patterns. This work reveals the accumulation of molecular, physiological and anatomical traits linked to behavioural divergence, and defines a powerful model for investigating nervous system evolution and speciation.

The genetic and neural basis by which animals adapt behaviourally to their ecological niche is largely unknown ${ }^{1,2}$. Insights have been gained from intraspecific variation in traditional model organisms, including anxiety behaviours in Mus musculus ${ }^{17}$ and exploration/ exploitation decisions in Caenorhabditis elegans ${ }^{18}$. Interspecific differences are more dramatic: Peromyscus mice species display variations in burrowing and parental care ${ }^{3,5}$, while the predatory nematode Pristionchus pacificus exhibits distinct feeding behaviours to C. elegans ${ }^{19}$. Defining the molecular basis of interspecific differences is challenging as it requires that species are comparable molecularly and anatomically, and genetically manipulatable.

Drosophilid flies are attractive models to investigate behavioural evolution: $D$. melanogaster offers deep neurobiological knowledge in a numerically-simple brain, and closely-related drosophilid species show distinct behaviours linked to their diverse ecologies ${ }^{20}$. Several of these behavioural traits have been correlated to anatomical and/or physiological changes in sensory or central pathways $4,11,13,15,21,22$. One remarkable drosophilid is the Seychelles endemic, D. sechellia, which shares a recent common ancestor with the cosmopolitan, ecological generalists D. melanogaster and D. simulans ${ }^{6,7}$ (Fig. 1a). D. sechellia has evolved extreme specialism for Morinda citrifolia "noni” fruit (Fig. 1a), and displays unique olfactory ${ }^{11-13,15,16}$, gustatory ${ }^{14}$, and reproductive behaviours ${ }^{8-10}$. Mapping approaches have located causal loci for some $D$. sechellia-specific traits within typically large genomic regions ${ }^{8,10}$, while candidate approaches have correlated chemosensory phenotypes with changes in its peripheral sensory pathways ${ }^{11,14,15}$.

Despite the potential $D$. sechellia presents for comparative neuroscience, investigations of $D$. sechellia's behaviours have been limited by the lack of genetic tools. Here we develop $D$. sechellia into a genetic model system, moving from genotypic/phenotypic correlations to test the role of genetic changes in behavioural evolution.

\section{Specific noni attraction of $D$. sechellia}

Noni-derived volatiles are likely the initial cues that guide D. sechellia host-seeking ${ }^{16}$. We used two assays to compare attraction of wild-type strains of $D$. sechellia, D. simulans and D. melanogaster to noni at distinct spatial scales (Fig. 1b,c, Extended Data Fig. 1): In a longrange, wind tunnel assay ${ }^{23}, D$. sechellia displayed higher attraction to noni than its sister species (Fig. 1b); in a short-range trap assay ${ }^{15}$, only $D$. sechellia exhibits marked noni preference (Fig. 1c). The level of attraction/preference of this species to noni juice (a more reproducible odour stimulus) was comparable to that for ripe fruit (Fig. 1b,c), concordant with their qualitatively similar odour bouquets (Fig. 1d, Extended Data Fig. 2a-b, Methods). Assays with other natural odour sources, as previously described in field studies ${ }^{24}$, confirmed the unique attractiveness of noni for D. sechellia (Extended Data Fig. 1a-e,g). 


\section{Noni-sensing olfactory pathways}

Drosophilids detect odours using olfactory sensory neurons (OSNs) in sensilla on their antennae and maxillary palps ${ }^{25}$. Most OSNs express a single Odorant Receptor (OR) or Ionotropic Receptor (IR) - which defines odour-tuning properties - along with an obligate co-receptor ${ }^{26-28}$. Neurons expressing the same tuning receptor converge onto a discrete glomerulus in the antennal lobe ${ }^{25}$. Electrophysiological analyses in $D$. sechellia have identified several OSN populations responding to individual noni odours ${ }^{11,13,15,29}$, but the global representation of the noni bouquet has not been examined.

We generated transgenic $D$. sechellia expressing GCaMP6f in the majority of OSNs under the control of Gal4 inserted at the Or co-receptor (Orco) locus (Extended Data Fig. 3). Using widefield imaging to compare this and an equivalent $D$. melanogaster line (Extended Data Fig. 4a-b), we did not detect novel noni-responsive olfactory channels in $D$. sechellia but rather quantitative differences in individual glomerular responses between species (Extended Data Fig. 4a). Two-photon calcium imaging highlighted two glomeruli (DM2 and VM5d) distinguished by their very high sensitivity to noni compared to grape juice in $D$. sechellia (Fig. 1e-f; Extended Data Fig. 4c-d). These glomeruli are innervated by OSNs housed in a common antennal basiconic sensillum class (ab3) that responds electrophysiologically to individual noni odours ${ }^{11,13}$.

\section{Genetic targeting of olfactory receptors}

For noni-sensitive olfactory channels, we determined their electrophysiological responses to noni odours (Fig. 2a-b, Extended Data Fig. 4e, 5-6) and mutated candidate olfactory receptors (Extended Data Fig. 5-6). In wild-type ab3 sensilla, the larger-spiking ab3A neuron responded most strongly to methyl esters while the smaller-spiking ab3B neuron was highly stimulated by 2-heptanone and 1-hexanol (Fig. 2a). In DsecOrco mutants, all of these responses were lost (Fig. 2a), indicating their dependence upon OR signalling.

D. melanogaster ab3A expresses the Or22a and Or $22 b$ genes ${ }^{30}$ while $D$. sechellia possesses only $\operatorname{Or} 22 a^{11}$. Targeted mutation of this locus abolished odour-evoked responses of ab3A, but not ab3B (Fig. 2a). The receptor in D. melanogaster ab3B is thought to be OR85b $\mathrm{b}^{25,31}$, but $\mathrm{DsecOr} 85 \mathrm{~b}$ mutant neurons retained some sensitivity to noni odours (Fig. 2a). Deletion of DsecOr $85 b$ and the neighbouring $\mathrm{DsecOr} 85 \mathrm{c}$, whose transcripts were detected in an antennal transcriptome ${ }^{32}$, led to complete loss of ab3B responses, arguing for partial receptor redundancy (Fig. 2a).

D. sechellia Ir75b neurons (in antennal coeloconic 3I (ac3I) sensilla) have evolved novel sensitivity to hexanoic acid ${ }^{15}$. Mutations in DsecIr $75 b$ or DsecIr8a (which encodes the acidsensing IR co-receptor ${ }^{27}$ ) led to selective loss of hexanoic and butyric acid responses in ac3I (Fig. 2b, Extended Data Fig. 6). Mutation of DsecOr35a (expressed in the paired neuron) diminished responses to all odours except these acids, in-line with the broad tuning of this receptor in D. melanogaster ${ }^{33}$. 


\section{ORs required for long-range attraction}

We used the receptor mutants to determine the behavioural role of individual olfactory pathways. In the long-range assay, DsecOrco exhibited no attraction to the odour source (Fig. 2c). Strikingly, both $\mathrm{DsecOr} 22 \mathrm{a}$ and $\mathrm{DsecOr} 85 \mathrm{c} / \mathrm{b}$ mutants displayed similarly strong defects (Fig. 2c). By contrast, DsecOr35a mutants were not impaired (Fig. 2c). Loss of IR8a also led to a significant decrease in long-range attraction in $D$. sechellia (Fig. 2c). This does not appear to be primarily due to defects in the hexanoic acid-sensing pathway, as DsecIr $75 b$ mutants had either no or milder defects than DsecIr8a mutants (Fig. 2c). Mutations in DsecIr64a, which is broadly-tuned to acids in $D$. melanogaster ${ }^{34}$ and responded to noni in $D$. sechellia (Extended Data Fig. 4f-g), had no effect on this behaviour.

In the short-range assay, DsecOrco mutant flies displayed reduced, but not abolished, preference for noni (Fig. 2d). Individual Or pathway mutants had very slight (DsecOr22a) or no (DsecOr85c/b, DsecOr35a) defects in this behaviour (Fig. 2d, Extended Data Fig. 7). Mutants for DsecIr8a or DsecIr $75 b$, but not DsecIr64a, displayed reduced preference, with notable frequent preference reversals in several trials (Fig. 2d). Orco/Ir8a double mutants, or antenna-less flies, displayed no noni preference (Fig. 2e, Extended Data Fig. 7c-d), indicating that this short-range behaviour depends upon multiple, partially redundant olfactory inputs. Consistent with these observations, individual noni odours promoted strong preference at short-range, while they triggered no or little flight attraction at long-range (Extended Data Fig. 7f-g) ${ }^{11,13,15}$. The relative contribution of individual channels to these behaviours may be related to their different detection thresholds (Extended Data Fig. 2c) and/or differential diffusion of cognate odours within each assay (Extended Data Fig. 2d-e).

\section{Tuning of OR22a impacts behaviour}

Given the crucial role of OR22a and OR85c/b in long-range attraction, we explored the evolution of these pathways. Or85c/b neurons displayed an indistinguishable sensitivity across species to their best agonist, 2-heptanone. By contrast, both $D$. sechellia and $D$. simulans Or22a/(b) neurons exhibited increased sensitivity to methyl hexanoate compared to D. melanogaster (Fig. 3a, Extended Data Fig. 8a) ${ }^{11,29}$. Broader profiling of Or22a/(b) neurons in the D. melanogaster species subgroup (Fig. 3b, Extended Data Fig. 8g) revealed that $D$. sechellia was the only species with selective, high sensitivity to methyl esters; others, including $D$. simulans, also responded to ethyl esters (Fig. 3b). This suggests that changes in tuning sensitivity and/or breadth of OR22a - but not OR $85 \mathrm{c} / \mathrm{b}$ - contribute to the differential behaviour of $D$. sechellia.

We next reintroduced DsecOr22a $a^{w t}$, DsimOr22a ${ }^{w t}$ or DmelOr22awt into the DsecOr22a endogenous locus (Extended Data Fig. 8b). DsecOr22a $a^{w t}$ and DmelOr $22 a^{w t}$ restored electrophysiological response profiles similar to native neuronal responses, indicating that the receptor is key for species-specific neuron tuning (Fig. 3c, upper part). DsimOr22awt conferred sensitivity to methyl but not ethyl esters (Fig. 3b-c, Extended Data Fig. 8h); genetic analysis in $D$. simulans indicated that detection of ethyl esters by the endogenous Or22a/b neurons depends upon the co-expressed OR22b (Extended Data Fig. 8c-f). 
Concordant with their physiological properties, DsecOR22a ${ }^{\text {wt }}$ and $D$ simOR $22 \mathrm{a}^{\mathrm{wt}}$ but not DmelOR $22 \mathrm{a}^{\mathrm{wt}}$, rescued long-range behavioural responses to almost wild-type levels (Fig. 3d). Reciprocally, expression of $D s e c \mathrm{OR} 22 \mathrm{a}^{\mathrm{wt}}$ in $\operatorname{Or} 22 \mathrm{a} / \mathrm{b}$ mutant neurons in $D$. melanogaster (Fig. 3e, upper part) conferred higher noni sensitivity and long-range attraction than DmelOR22a ${ }^{\text {wt }}$ (Fig. 3f, Extended Data Fig. 8i, 9a).

\section{Molecular basis of OR22a tuning changes}

We next sought the molecular basis of OR22a's tuning differences. Expression of chimeric versions of DsecOR22a ${ }^{\text {wt }}$ and DmelOR22a ${ }^{\text {wt }}$ in $D$. melanogaster Or22a/b neurons (Extended Data Fig. 9c) indicated that high-sensitivity and selectivity for methyl esters were determined by the N-terminal 100 amino acids of DsecOR22a (chimera C) (Extended Data Fig. 9b-c,e). Within these, three positions (I45, I67 and M93) differ between Dme/OR22a and its orthologues in species displaying narrowed tuning for methyl esters (Extended Data Fig. 9b). Exchange of these residues (Dme/OR22a $\mathrm{a}^{\mathrm{I45V}, \mathrm{I67M}, \mathrm{M} 93 \mathrm{I}}\left(\right.$ Dme/OR22a $\left.^{\text {triple }}\right)$ ) narrowed responsiveness to methyl esters, similar to DsecOR22a ${ }^{\text {wt }}$ (Fig. 3c,e (lower parts), Extended Data Fig. 9d,f). Individual mutations revealed that Dme/OR22a ${ }^{\mathrm{M} 93 \mathrm{I}}$ most closely recapitulated the higher sensitivity of this receptor to methyl esters over ethyl esters (Fig. 3c,e, (lower parts), Extended Data Fig. 9d-g). Conversely, DsecOR22a ${ }^{193 \mathrm{M}}$ exhibited broadened sensitivity to both ester classes (Fig. 3c (lower part), Extended Data Fig. 9g).

In the long-range olfactory behaviour assay, expression of DmelOR22a ${ }^{\text {triple }}$ in D. sechellia Or22a neurons restored $D$. sechellia-like attraction to noni, while both DmelOR22 $\mathrm{a}^{\mathrm{M} 93 \mathrm{I}}$ and DsecOR22a ${ }^{193 M}$ displayed levels of attraction intermediate between those of the wild-type receptor rescues (Fig. 3d). Similarly, expression of DmelOR22a ${ }^{\text {triple }}$ in D. melanogaster conferred noni attraction at equivalent levels to DsecOR22a ${ }^{\text {wt }}$, while DmelOR22a ${ }^{\text {M93I }}$ supported intermediate attraction levels (Fig. 3f). These results provide evidence that the molecular differences in OR22a orthologues contribute to species-specific olfactory behaviours.

\section{Sensory representation of Or22a}

The functional similarity of OR22a orthologues in D. sechellia and D. simulans (Fig. 3c-d) indicates that additional changes occurred during speciation of $D$. sechellia. Concordant with ab3 sensilla counts ${ }^{11,29}, D$. sechellia exhibits a three-fold increase in the number of Or22a neurons (recapitulated in rescue experiments, Extended Data Fig. 10a) and the paired Or85c/b neurons, but not several other neuron classes (Fig. 4a-b, Extended Data Fig. 5d, 10b and $\left.{ }^{15}\right)$ ).

To analyse OSN projections in D. sechellia, we inserted Gal4 at the corresponding receptor loci, and combined these with UAS-GCaMP6f as anatomical marker (Extended Data Fig. 3). Extending single-neuron dye-filling analyses ${ }^{11,13,15}$, OSN glomerular innervation patterns were indistinguishable between D. sechellia and D. melanogaster (Fig. 4c, Extended Data Fig. 3c-d). However, the glomerular targets of Or22a and Or85c/b neurons (DM2 and VM5d, respectively) were nearly doubled in volume in $D$. sechellia compared to both other species (Fig. 4d) ${ }^{11,13}$. 


\section{Differences in Or22a circuit wiring}

To visualise higher-order elements of the Or22a pathway, we combined a pan-neuronal driver (Extended Data Fig. 10c) with a photoactivatable-GFP transgene to selectively photolabel DM2 projection neurons (PNs). Analysis with analogous genetic reagents in $D$. melanogaster - as well as targeted electroporation of a lipophilic dye ${ }^{35}$ into this glomerulus in $D$. simulans, $D$. sechellia and $D$. melanogaster-permitted cross-species comparisons. Two DM2 projection neurons (PNs) were consistently labelled in all three drosophilids (Fig. $4 \mathrm{e})$.

PNs innervate the mushroom body (required for learning/memory) and the lateral horn (implicated in innate olfactory responses) ${ }^{36}$. Within the former, the number and arrangement of PN axonal branches were similar between species (Fig. 4e). In the lateral horn, global anatomy was conserved, with the main tract bifurcating into dorsal and ventral branches. However, dorsal to the bifurcation, $D$. sechellia DM2 PNs had a prominent branch innervating an area not targeted by the homologous $D$. melanogaster or $D$. simulans neurons (Fig. 4e-f). Using successive photo- and dye-labelling to visualise single DM2 PNs in $D$. sechellia and $D$. melanogaster, we confirmed quantitatively the presence of a $D$. sechelliaspecific branch (Fig. 4g); this was also detected in DsecOr22a mutant animals (Extended Data Fig. 10d-e), indicating its independence of sensory input. These data raise the possibility that $D$. sechellia-specific central circuit changes form part of its olfactory specialisation towards noni.

\section{Discussion}

We have developed $D$. sechellia as a model to link genetic and neural circuit changes to behaviours relevant for its ecology. The characterisation of the Or22a pathway and comparison of this circuit's functional and structural properties across closely-related species provides several insights into behavioural evolution (Fig. 4h).

The Or22a allele transfer experiments provide evidence that olfactory receptor tuning contributes to species-specific odour-evoked behaviour. Our definition of determinants of OR22a re-tuning also informs the molecular basis of odour/receptor interactions. When mapped onto a presumed homologous ORCO structure ${ }^{37}$, the key change (M93I) falls within a putative ligand-binding pocket, and may be a "hot-spot" for functional evolution (Extended Data Fig. 11a-c).

Although functional differences in this receptor are important, they cannot explain the behavioural differences of $D$. sechellia and $D$. simulans, as these receptors are interchangeable for supporting noni attraction. We note that the native Or22a/(b) neuron responses of these species are not identical (Fig. 3a-b); loss of Or $22 b$ in $D$. sechellia led to a narrowed (and possibly slightly increased) sensitivity to methyl esters, which could be behaviourally relevant. The $D$. sechellia-specific expansion of this neuron population is a likely key additional evolutionary innovation, although it is insufficient alone to restore $D$. sechellia-like host attraction when expressing DmelOR22a. The difference in D. sechellia PN axon projections suggests that central circuit connectivity changes form part of this 
species' adaptation to noni. Future studies are necessary to understand the genetic bases and behavioural significance of these neuroanatomical differences.

The critical role of OR22a in host attraction in $D$. sechellia may account for the rapid molecular evolution of this locus (Extended Data Fig. 11d-h) ${ }^{38-40}$. D. erecta, a specialist on Pandanus fruit, also exhibits expansion of this OSN population ${ }^{21}$. However, a second noniadapted drosophilid, D. yakuba mayottensis ${ }^{41}$ does not share the same receptor or OSN number changes we describe (Extended Data Fig. 11i-m) implying an independent evolutionary solution to locate a common host fruit.

Finally, other olfactory channels are important for noni attraction. These include Or85c/b neurons, which have conserved physiology while increasing in number in D. sechellia, and Ir75b neurons, which have both changed in function and number while apparently preserving partner PN projections ${ }^{15}$. Future application of the D. sechellia genetic toolkit should offer further fundamental insights into how genes and neurons control behaviour and enable evolution of novel traits.

\section{Methods}

\section{Data reporting}

Preliminary experiments were used to assess variance and determine adequate sample sizes in advance of acquisition of the reported data. Several experiments were carried out repeatedly because they served as controls for different genetic manipulations. In particular, we ran wild-type controls in parallel with mutant analyses in behavioural assays and therefore replicated them multiple times. In the wind tunnel assay the number of possible samples per day is rather low, leading to testing of flies of different genotypes on different days. In these cases experiments were started at the same time of the day under the stringently controlled conditions of temperature, humidity, light, and age of flies. For electrophysiological recordings, data were collected from multiple flies on multiple days in randomised order interleaving wild-type and mutant genotypes. Within datasets the same odour dilutions were used for acquisition of the dataset. In all cases the results were reliable and robust over the course of the many years it took to complete this study. For olfactory trap assays, the experiments were conducted with the experimenter blinded to the genotype. The experimenter was not blinded to the genotype of flies in the wind tunnel assay or physiological experiments. All replicates are biological replicates.

\section{Volatile collection, gas chromatography and mass spectrometry}

Volatiles were collected from $1 \mathrm{ml}$ of fruit juice or $13 \mathrm{~g}$ of noni fruit at different ripening stages in capped $15 \mathrm{ml}$ glass vials with poly-tetra-fluoroethylene-lined silicone septa (Sigma, 23242-U). After penetrating the septum of the cap with a Solid Phase Microextraction (SPME) fibre holder, the SPME fibre (grey hub plain, coated with 50/30 $\mu \mathrm{m}$ divinylbenzene/carboxen on polydimethylsiloxane on a StableFlex fibre (Sigma, 57328-U)) was exposed to the headspace of each vial for $30 \mathrm{~min}$ at room temperature. For collection of headspaces in the trap assay, a single noni juice trap was placed in the arena and odours were collected with SMPE for $5 \mathrm{~min}$ at $0 \mathrm{~h}, 5 \mathrm{~h}$ and $10 \mathrm{~h}$ after placement. For collection of 
headspaces in the wind tunnel assay, samples were captured with SPME for $10 \mathrm{~min}$ at the landing platform and the release platform directly after noni juice application. After each odour collection, the SPME fibre was retracted and immediately inserted into the inset of a Gas Chromatography-mass spectrometry (GC-MS) system (Agilent 7890B fitted with MS 5977 A unit) for desorption at $260^{\circ} \mathrm{C}$ in split mode (split ratio 100:1). The $\mathrm{GC}$ was operated with a HP-INNOWax column (Agilent 19091N-133UI). Samples were injected at an initial oven temperature of $50^{\circ} \mathrm{C}$; this temperature was held for $1 \mathrm{~min}$ and gradually increased $\left(3^{\circ} \mathrm{C}\right.$ $\left.\min ^{-1}\right)$ to $150^{\circ} \mathrm{C}$ before holding for $1 \mathrm{~min}$. Subsequently, the temperature was increased $\left(20^{\circ} \mathrm{C} \mathrm{min}^{-1}\right)$ to $260^{\circ} \mathrm{C}$ and held for $5 \mathrm{~min}$. The MS-transfer-line was held at $260^{\circ} \mathrm{C}$, the MS source at $230^{\circ} \mathrm{C}$, and the MS quad at $150^{\circ} \mathrm{C}$. MS spectra were taken in EI-mode $(70 \mathrm{eV})$ in a $29-350 \mathrm{~m} / \mathrm{z}$ range. Between different collections, the SPME fibre was conditioned at $270^{\circ} \mathrm{C}$ for $15 \mathrm{~min}$. All chromatograms were processed using MSD ChemStation F.01.03.2357 software. Volatile compounds were identified using the NIST library and matched to standards of the Max-Planck-Institute for Chemical Ecology library. For quantification, peak areas were measured for 3 replicates for each sample. Note that SPME allows only for a qualitative analysis of odour compositions as well as for an estimate of changing ratios of odours across different samples. Vapour pressure values for hexanoic acid, methyl hexanoate and 2-heptanone were described previously (www.thegoodscentscompany.com) ${ }^{42,43}$.

\section{Drosophila strains}

Drosophila stocks were maintained on standard wheat flour/yeast/fruit juice medium under a $12 \mathrm{~h}$ light: $12 \mathrm{~h}$ dark cycle at $25^{\circ} \mathrm{C}$. For all $D$. sechellia strains, a few $\mathrm{g}$ of Formula 4-24® Instant Drosophila Medium, Blue (Carolina Biological Supply Company) soaked in noni juice (nu3 $\mathrm{GmbH}$ ) were added on top of the standard food. Wild-type Drosophila strains are described in the corresponding figure legends or Supplementary Table 2. These strains do not show intraspecific sequence variation in OR22a or IR75b for known odour specificitydetermining residues (Extended Data Fig. 12), suggesting that other polymorphisms (or nongenetic factors) underlie the observed minor intraspecific behavioural differences (Fig. 1b,c). The mutant and transgenic lines used and generated in this study are listed in Supplementary Table 2 .

\section{CRISPR/Cas9-mediated genome engineering}

sgRNA expression vectors: for expression of single sgRNAs, oligonucleotide pairs (Supplementary Table 3) were annealed and cloned into BbsI-digested $p C F D 3-d U 6-3 g R N A$ (Addgene \#49410), as described ${ }^{44}$. To express multiple sgRNAs from the same vector backbone, oligonucleotide pairs (Supplementary Table 4) were used for PCR and inserted into $p C F D 5$ (Addgene \#73914) via Gibson Assembly, as described ${ }^{45}$.

Donor vectors for homologous recombination: to generate an eGFP-expressing donor vector ( $p H D$-Stinger-attP), the fluorophore was excised from pStinger $^{46}$ with Ncoll HpaI and used to replace the DsRed sequence in NcoIl Hpal-digested $p H D$-DsRed-attP(Addgene plasmid $\# 51019)^{47}$. Homology arms (1-1.6 kb) for individual target genes were amplified from $D$. sechellia (Drosophila Species Stock Center [DSSC] 14021-0248.07), D. simulans (DSSC 14021-0251.195) or D. melanogaster (Research Resource Identifier Database:Bloomington Drosophila Stock Center [RRID:BDSC]_58492) genomic DNA and inserted either into 
pHD-DsRed-attP or $p H D$-Stinger-attPvia restriction cloning. Details and oligonucleotide sequences are available from the corresponding authors upon request.

Transgenic source of Cas9: pBac(nos-Cas9,3XP3-YFP) (gift of D. Stern) was integrated into D. sechellia (DSSC 14021-0248.07) via piggyBac transgenesis. The insertion was mapped to the fourth chromosome using TagMap ${ }^{48}$.

\section{Transgene construction}

Oligonucleotides for each cloning step are listed in Supplementary Table 5.

attB-nSyb-Gal4,miniW: $1.9 \mathrm{~kb}$ upstream sequence of the neuronal Synaptobrevin ( $n S y b)$ gene were amplified from $D$. sechellia genomic DNA (DSSC 14021-0248.07) and inserted into $p$ Gal4att $B^{49}$ via restriction cloning using $\mathrm{NotI}$ and $\mathrm{KpnI}$.

attB-Gal4,3XP3-Stinger: we first generated an eGFPnls-SV4O fragment via PCR (using $p H D$-Stinger-attP as template) and fused it to a minimal attB40 site $^{50,51}$ before insertion into pCR-Blunt II-TOPO (Thermo Fisher). We added a 3XP3-Stinger fragment amplified from $p H D$-Stinger via restriction cloning using $E c o R V$ and SalI. Subsequently, we placed a $l o x P$ site downstream of the initial $S V 40$ sequence via oligonucleotide annealing and SpeII KpnI restriction cloning, to produce $p C R$-TOPO-loxP-attB40-eGFPnlsSV40rev-3XP3:Stinger. We replaced the eGFPnls-SV4O sequence with an hsp70-Gal4-SV4O fragment via PCR amplification of the vector backbone and Gal4 from $p G a l 4 a t t B^{49}$ and subsequent Gibson Assembly resulting in attB-Gal4,3XP3-Stinger.

attB-Or22a ${ }^{w t}, 3 X P 3-S t i n g e r$ in the $p C R$-TOPO-loxP-attB40eGFPnlsSV4Orev-3XP3-Stinger plasmid described above, the eGFPnlsSV4O fragment was flanked by EcoRV and $S a l I$ sites, which were used to integrate the D. sechellia or D. melanogaster Or22a ORF+3'UTR after PCR amplification from cDNA, or the $D$. simulans Or22a ORF+3'UTR (synthesised by Eurofins Genomics), to produce attB-DsecOr22a ${ }^{w t}, 3 X P 3-$ Stinger, attB-DmelOr22a ${ }^{w t}, 3 X P 3-$ Stinger, and attB-DsimOr22a ${ }^{w t}, 3 X P 3-S t i n g e r$, respectively.

Or22a chimeras: chimeric sequences of $D$. sechellia and D. melanogaster Or22a were generated by PCR amplification and fusion using the respective species' Or22a gene templates. After subcloning into $p C R$-Blunt II-TOPO and sequence confirmation, the chimeras were integrated into $p C R-T O P O$ lox $P$ attB4OeGFPnlsSV4Orev-3XP3-Stinger via restriction cloning.

Or22a site-directed mutant constructs: point mutations were introduced via site directed mutagenesis following standard procedures.

attB-UAS-constructs: DsecOr22awt, DmelOr22a ${ }^{w t}$, DmelOr22a ${ }^{\text {triple }}$ and DmelOr22a ${ }^{M 93 I}$ were amplified by PCR incorporating flanking EcoRI and SalI restriction sites using the constructs described above as template, and integrated into the EcoRI/XhoI-digested pUAST-attB ${ }^{52}$.

pDONR221-MCS: a $p D O N R 221$ entry vector carrying a multiple cloning site (MCS) was generated by amplification of the MCS of $p C R$-Blunt II-TOPO incorporating flanking 
attB1/2 sites and integration of the PCR fragment into $p D O N R 221$ via a BP reaction (Gateway, Thermo Fisher Scientific).

pDONR221-DsimOr22a: a $5.7 \mathrm{~kb}$ promoter region upstream of the Or22a start codon was amplified from $D$. simulans (DSSC 14021-0251.195) genomic DNA, subcloned into $p C R$ Blunt II-TOPO and transferred to $p D O N R 221-M C S$ via BamHI/EcoRV restriction cloning.

pDEST-Hemmar-eGFPnls: the eGFPnls fragment of pStinger ${ }^{46}$ was amplified by PCR incorporating $\mathrm{XhoI}$ and $\mathrm{SpeI}$ restriction sites and integrated into the XhoI/Xbal-digested vector $p D E S T$-Hemmar $G^{53}$ to replace $e G F P$.

pDsimOr22a-eGFPnls: pDONR221-DsimOr22a and pDEST-Hemmar-eGFPnls were combined using LR recombination (Gateway, Thermo Fisher Scientific).

\section{Drosophila microinjections}

Transgenesis of $D$. sechellia, D. simulans and D. melanogaster was performed in-house following standard protocols (http://gompel.org/methods), except for DsimOr22a-GFPnls (generated by Rainbow Transgenic Flies Inc.). For the D. sechellia egg-laying agar plates, we replaced grape juice with noni juice and added on the surface a few $g$ of Formula 4-24® Instant Drosophila Medium, Blue (Carolina Biological Supply Company) soaked in noni juice (nu3 $\mathrm{GmbH}$ ). Embryos were manually selected for the appropriate developmental stage prior to alignment and injection. For piggyBac transgenesis, we co-injected piggyBac vector (300 $\mathrm{ng} \mathrm{\mu l}^{-1}$ ) and piggyBac helper plasmid ${ }^{54}\left(300 \mathrm{ng} \mathrm{\mu l}^{-1}\right.$ ). For CRISPR/Cas9-mediated homologous recombination, we injected a mix of an sgRNA-encoding construct (150 ng $\mathrm{\mu l}^{-1}$ ), donor vector $\left(400 \mathrm{ng}^{-1} \mathrm{l}^{-1}\right.$ ) and $\mathrm{pHsp} 70-$ Cas $9\left(400 \mathrm{ng} \mathrm{\mu l}^{-1}\right.$ ) (Addgene \#45945) ${ }^{55}$. The DsRed fluorescent marker was destroyed in DsecnSyb-Gal4 and DsecUAS-C3PA-GFP via

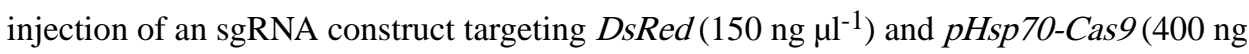
$\left.\mu^{-1}\right)$. Injections into Dsecnos-Cas 9 were of a mix of an sgRNA construct $\left(150 \mathrm{ng} \mathrm{\mu l}^{-1}\right)$ and donor vector $\left(500 \mathrm{ng}^{-1} \mathrm{l}^{-1}\right)$. Site-directed integration into att $P$ sites was achieved by coinjection of an attB-containing vector $\left(400 \mathrm{ng}^{-1} \mathrm{l}^{-1}\right)$ and either $p 3 x P 3-E G F$.vas-int.NLS $\left(400 \mathrm{ng}^{-1} \mathrm{l}^{-1} \text { ) (Addgene } \# 60948\right)^{56}$ or $p B S 130$ (encoding phiC31 integrase under control of a heat shock promoter (Addgene \#26290) ${ }^{57}$ ). All concentrations are given as final values in the injection mix.

\section{Wind tunnel assay}

Long-range attraction experiments were performed in a wind tunnel as described previously ${ }^{23}$ with a flight arena of $30 \mathrm{~cm}$ width, $30 \mathrm{~cm}$ height and $100 \mathrm{~cm}$ length. The airstream in the tunnel $\left(0.3 \mathrm{~m} \mathrm{~s}^{-1}\right)$ was produced by a fan (Fischbach $\mathrm{GmbH}$, Neunkirchen, Germany), and filtered through an array of four activated charcoal cylinders $(14.5 \mathrm{~cm}$ diameter $\times 32.5 \mathrm{~cm}$ length; Camfil, Trosa, Sweden). The wind tunnel was maintained within a climate chamber at $25^{\circ} \mathrm{C}$ and $50-55 \%$ relative humidity under white light. Flies were starved for approximately $20 \mathrm{~h}$; to ensure the flight ability of assayed animals, flies were first released into a mesh cage $(50 \times 50 \times 50 \mathrm{~cm}$, maintained at the same conditions as the wind tunnel) and females escaping from the food vial were collected with an aspirator. For each assay, ten 4-6 day-old females were released from a plastic tube (with a mesh covering 
one end and the open end facing the landing platform) fixed horizontally in the centre of the first 5-10 $\mathrm{cm}$ of the downwind end of the tunnel. The landing platform was built by a filter paper $(3 \times 3 \mathrm{~cm})$ charged with either $100 \mu$ of juice (noni $(\mathrm{Nu} 3 \mathrm{GmbH})$, grape (Beutelsbacher Fruchtsaftkelterei), pineapple (Andros), mango (Migros)), apple cider vinegar (Migros) or $\sim 100 \mu \mathrm{l}$ of homogenised ripe fruit (noni, fig) and fixed on a metal holder. Fruit homogenisation was performed by blending $10 \mathrm{mg}$ of ripe fruit in $20 \mathrm{ml}$ of distilled water, fruit particles were pelleted by centrifugation and the supernatant harvested for experiments. In two-choice assays two identical landing platforms were positioned with equal distance $(7.5 \mathrm{~cm})$ from the centre of the air stream alternating the position of noni fruit and apple cider vinegar between assays. The fly tube was placed within the centre of the airstream and $85 \mathrm{~cm}$ downwind of the odour source. An experimenter observed the landing platform(s) for the entire duration of the assay; flies arriving and staying on the landing platform(s) within the first 10 min after release were counted.

\section{Olfactory trap assay}

The two-choice olfactory trap assay was performed essentially as described ${ }^{15}$. For each experiment, traps contained either $300 \mu \mathrm{l}$ of juice (noni (Nu3 GmbH), grape (Beutelsbacher Fruchtsaftkelterei), pineapple (Andros), mango (Migros)) or apple cider vinegar (Migros). When using fruits as stimuli (noni, grape, papaya, banana, fig), ripe fruits were peeled (banana, papaya) or used whole, homogenised with pestles, and each trap was filled with a spatula of the mix (to $\sim 300 \mu \mathrm{l}$ ). Single odours (see below for CAS numbers) were used at a $10^{-2}$ dilution in grape juice. Triton X-100 (final concentration $0.2 \%$ ) was added to all traps containing single odours and respective control traps to drown trapped flies. 25 fed, mated, ice-anesthetised female flies (3-5 day-old) were used for each experiment. D. sechellia flies were transferred to standard food without noni supplement $24 \mathrm{~h}$ prior to the start of the assay, unless stated otherwise. The distribution of flies was scored after $24 \mathrm{~h}$ at $25^{\circ} \mathrm{C}$ under red light at $60 \%$ relative humidity; experiments with $>25 \%$ dead flies in the arena after $24 \mathrm{~h}$ were discarded. The attraction index was calculated as follows: (number of flies in treatment (e.g., noni juice) trap - number of flies in control (e.g., grape juice) trap)/number of trapped and untrapped flies alive. For the trap assay quantifications in Fig. 2e, all untrapped flies (including those that died during the assay) were counted due to the high mortality rate in these experiments (Extended Data Fig. 7c).

The multiple-choice olfactory trap assay was performed using eight traps containing mango, pineapple, noni and grape juices, apple cider vinegar and fig, banana and papaya fruits. Traps were placed equidistantly in a circle in random order for each experiment and conditions were as described for the two-choice assay. The percentage of flies per trap was calculated as follows: (number of flies in trap/number of trapped and untrapped flies alive) $\times 100$. Experiments with $>25 \%$ dead flies in the arena after $24 \mathrm{~h}$ were discarded.

\section{Two-photon calcium imaging}

Flies were mounted and dissected as previously described ${ }^{58}$, and images were acquired using a commercial upright two-photon microscope (Zeiss LSM 710 NLO). In detail, an upright Zeiss AxioExaminer Z1 was fitted with a Ti:Sapphire Chameleon Ultra II infrared laser (Coherent) as excitation source. Images were acquired with a 20x water-immersion 
objective (W Plan-Apochromat 20×; NA 1.0, VIS-IR DIC), with a resolution of $128 \times 128$ pixels $\left(1.1902\right.$ pixels $\left.\mu \mathrm{m}^{-1}\right)$ and a scan speed of $12.6 \mu$ pixel $^{-1}$. The excitation wavelength was set to $920 \mathrm{~nm}$ at a laser output of 64.1-70.2 $\mathrm{mW}$ measured at the exit of the objective. Emitted light was filtered with a 500-550 nm band-pass filter, and photons were collected by an external non-descanned detector. Each measurement consisted of 50 images acquired at $4.13 \mathrm{~Hz}$, with stimulation starting $\sim 5 \mathrm{~s}$ after the beginning of the acquisition and lasting for 1 s. Fly antennae were stimulated using a custom-made olfactometer as previously described $^{59}$, with minor modifications. In brief, the fly's antennae were permanently exposed to air flowing at a rate of $1.51 \mathrm{~min}^{-1}$ and with $55 \%$ relative humidity obtained by combining a main stream of humidified room air $\left(0.51 \mathrm{~min}^{-1}\right)$ and a secondary stream (1 1 $\mathrm{min}^{-1}$ ) of normal room air. Both air streams were generated by vacuum pumps (KNF Neuberger AG) and the flow rate was controlled by two independent rotameters (Analyt). The secondary air stream was guided through either an empty $2 \mathrm{ml}$ syringe or, to generate an odour pulse, a $2 \mathrm{ml}$ syringe containing $20 \mu \mathrm{l}$ of odour or solvent on a small cellulose pad (Kettenbach $\mathrm{GmbH}$ ). Solvents were either double-distilled water (for noni juice and apple cider vinegar) or paraffin oil (for methyl hexanoate (CAS 106-70-7), 2-heptanone (CAS 110-43-0), 2,3-butanedione (CAS 431-03-8), ethyl propionate (CAS 105-37-3) and 1hexanol (CAS 111-27-3)). To switch between control air and odour stimulus delivery, a three-way magnetic valve (The Lee Company, Westbrook, CT) was controlled using Matlab via a VC6 valve controller unit (Harvard Apparatus). Data were processed using Fiji ${ }^{60}$ and custom written scripts in Matlab and R as previously described ${ }^{59}$. Since bleaching was very strong at the beginning of each acquisition, the first $1.5 \mathrm{~s}$ were not considered for the analysis, and bleach correction was not applied. Colour-coded images and boxplots show the peak response calculated as the mean relative change in fluorescence $(\% \Delta \mathrm{F} / \mathrm{F})$ of three frames around the maximum during frames 19-30.

\section{Widefield calcium imaging}

Flies were mounted and dissected as previously described ${ }^{58}$. Images were acquired with a CCD camera (CoolSNAP-HQ2 Digital Camera System) mounted on a fluorescence microscope (upright fixed stage Carl Zeiss Axio Examiner D1) equipped with a 20× waterimmersion objective (W Plan-Apochromat 20x; NA 1.0, VIS-IR DIC, Extended Data Fig. 4a) or 40× water-immersion objective (W Plan-Apochromat 40×; NA 1.0 VIS-IR DIC, Extended Data Fig. 4f). Excitation light of $470 \mathrm{~nm}$ was produced with an LED light (Cool LED pE-100, VisiChrome, intensity 1.8-3.9\%). Light was guided through a filter block consisting of a 450-490 nm excitation filter, a dichroic mirror (T495LP), and a 500-550 nm emission filter (Chroma ET). Binned image size was $400 \times 300$ pixels (Extended Data Fig. 4a) or $266 \times 200$ pixels (Extended Data Fig. 4f) on the chip, corresponding to $465 \times 349 \mu \mathrm{m}$ (Extended Data Fig. 4a) or 149×112 $\mu \mathrm{m}$ (Extended Data Fig. 4f) in the preparation. Exposure time varied between 30-100 ms to adjust for different basal fluorescence values across preparations. Films (12.5 s duration) were recorded with an acquisition rate of $4 \mathrm{~Hz}$. Metafluor software (Visitron) was used to control the camera, light, data acquisition and onset of odour stimulation. Odour stimulation and data analysis were otherwise performed as described for two-photon calcium imaging. 


\section{Electrophysiology}

Single sensillum electrophysiological recordings were performed as described previously ${ }^{61}$.

Noni and grape juice were purchased from $\mathrm{Nu} 3(\mathrm{nu} 3 \mathrm{GmbH})$ and Beutelsbacher

(Beutelsbacher Fruchtsaftkelterei $\mathrm{GmbH}$ ) and chemicals of the highest purity available from Sigma-Aldrich. Odorants were used at $10^{-2}(\mathrm{v} / \mathrm{v})$ in all experiments unless noted otherwise in the figures or figure legends. Solvents were either double-distilled water (for noni juice, butyric acid (CAS 107-92-6), hexanoic acid (CAS 1821-02-9)) or paraffin oil (for octanoic acid (CAS 124-07-2), methyl butanoate (CAS 623-42-7), methyl hexanoate (CAS 106-70-7), methyl octanoate (CAS 111-11-5), ethyl butanoate (CAS 105-54-4), ethyl hexanoate (CAS 123-66-0), ethyl octanoate (CAS 106-32-1), 2-heptanone (CAS 110-43-0), 1-hexanol (CAS 111-27-3) and 3-buten-1-ol (CAS 627-27-0)). Odours for sensilla stimulation were used for a maximum of five consecutive trials. If noni juice or noni fruit extract (see Wind tunnel assay section for details) were used, odours were renewed after two stimulations or, for $10^{-4}$, $10^{-3}$ and $10^{-2}$ dilutions, after each stimulation. Corrected responses were calculated as the number of spikes in a $0.5 \mathrm{~s}$ window at stimulus delivery ( $200 \mathrm{~ms}$ after stimulus onset to take account of the delay due to the air path) subtracting the number of spontaneous spikes in a $0.5 \mathrm{~s}$ window $2 \mathrm{~s}$ before stimulation, multiplied by two to obtain spikes $\mathrm{s}^{-1}$. The solventcorrected responses shown in the figures were calculated by subtracting from the response to each diluted odour the response obtained when stimulating with the corresponding solvent. Recordings were performed on a maximum of three sensilla per fly. Exact $\mathrm{n}$ and mean spike counts for all experiments are provided in Supplementary Data Table 7.

\section{Immunohistochemistry}

Fluorescent RNA in situ hybridisation using digoxigenin- or fluorescein-labelled RNA probes and immunofluorescence on whole-mount antennae were performed essentially as described $^{59,62}$. D. sechellia probe templates were generated by amplification of regions of genomic DNA (DSSC 14021-0248.07) using primer pairs listed in Supplementary Data Table 6; these were cloned into $p C R$-Blunt II-TOPO and sequenced. D. sechellia OR22a antibodies were raised in rabbits against the peptide epitope PHISKKPLSERVKSRD (amino acids 7-22), affinity-purified (Proteintech Group Inc) and diluted 1:250. Other antibodies used were: guinea pig a-IR75b (RRID:AB_2631093) ${ }^{63} 1: 200$, rabbit a-IR64a (RRID:AB_2566854) $)^{34} 1: 100$, rabbit a-ORCO ${ }^{28} 1: 200$, guinea pig a-IR8a (RRID:AB_2566833) ${ }^{27} 1: 500$, rabbit a-IR25a (RRID:AB_2567027) 1:50064, rabbit a-GFP 1:500 (Invitrogen). Immunofluorescence on adult brains was performed as described ${ }^{65}$ (except for the $D$. sechellia reference brain samples; see below) using mouse monoclonal antibody nc82 1:10 (Developmental Studies Hybridoma Bank), rat monoclonal a-Elav 1:10 (Developmental Studies Hybridoma Bank) and rabbit a-GFP 1:500 (Invitrogen). Alexa488-, Cy3- and Cy5-conjugated goat a-guinea pig, goat a-mouse, goat a-rat and goat a-rabbit IgG secondary antibodies (Molecular Probes; Jackson Immunoresearch) were used at 1:500.

\section{D. sechellia reference brain}

D. sechellia (DSSC 14021-0248.07) brains (2-7 day-old animals) were stained with nc82 and imaged as described ${ }^{66}$. From 88 female brains imaged, 26 high-quality confocal stacks of the midbrain were selected for averaging on a selected "seed" brain, essentially as 
described $^{67,68}$. Similarly, a male reference brain (not shown here) was constructed, using 20 high-quality confocal stacks (from 87 initially imaged). Reciprocal bridging registrations between $D$. melanogaster and $D$. sechellia references brains were also generated to permit comparison of homologous neurons within a common template, essentially as described ${ }^{68}$. The reference brains ( $D s e c F$ and $D s e c M$ ), bridging registrations and associated code are available for download via http://jefferislab.org/si/auer2019.

\section{Image acquisition and processing}

Confocal images of antennae and brains were acquired on an inverted confocal microscope (Zeiss LSM 710) equipped with an oil immersion 40× objective (Plan Neofluar 40× Oil immersion DIC objective; 1.3 NA), unless stated otherwise. Images were processed in Fiji60. $D$. sechellia brains were imaged and registered to a $D$. sechellia reference brain using the Fiji CMTK plugin (https://github.com/jefferis/fiji-cmtk-gui), as described ${ }^{69}$. For segmentation of individual glomeruli of the antennal lobe, glomerular identity was confirmed by location and labelling with Gal4 reporters (Extended Data Fig. 3) and segmentation performed using Amira 6.5 (Thermo Fisher Scientific). Glomerular volumes were calculated following segmentation with the Segmentation Editor plugin of Fiji using the 3D Manager Plugin. OSN numbers were counted using the Cell Counter Plugin in Fiji or Imaris (Bitplane). Projection neuron morphologies were reconstructed and measured in neuTube $1.0 \mathrm{z}^{70}$.

\section{Projection neuron labelling}

Photoactivation was performed as described ${ }^{35}$ on 3-5 day-old female flies. Brains were dissected in saline ${ }^{71}$ (low carbonate; $2 \mathrm{mM} \mathrm{Mg}^{2+} \mathrm{pH}$ 7.2) and treated with collagenase (2 $\mathrm{mg} \mathrm{ml}^{-1}, 45 \mathrm{~s}$ ). Dissected brains were initially imaged at $925 \mathrm{~nm}$ to identify the DM2 glomerulus based on anatomical position. Photoactivation was achieved through multiple cycles of exposure to $710 \mathrm{~nm}$ laser light with a $15 \mathrm{~min}$ rest period between each cycle to allow diffusion of the photoactivated fluorophore within the neuron. Photoactivation and imaging was performed on an Ultima two-photon laser scanning microscope (Bruker) equipped with galvanometers driving a Chameleon XR laser (Coherent). Emitted photons were collected with a GaAsP photodiode detector (Bruker) or a PMT detector through a $60 \times$ objective (Olympus 60× water immersion; 0.9 NA).

PN dye-fillings were performed as described ${ }^{35}$ with some modifications. Brains were dissected in saline, briefly treated with collagenase $\left(2 \mathrm{mg} \mathrm{ml}^{-1}, 45 \mathrm{~s}\right)$, washed and pinned with fine tungsten wires to a Sylgard sheet (World Precision Instruments) in a $35 \mathrm{~mm}$ Petri dish (Falcon) filled with saline. Pulled glass electrodes were backfilled with Texas Red Dextran (3000, lysine fixable, Thermo Scientific). The electrode was targeted to the DM2 glomerulus using as a guide either basal expression of the pan-neuronal, photoactivatable GFP (D. sechellia and D. melanogaster) or DM2-specific labelling (D. simulans, i.e., the Or22a-GFPnls strain, in which trace GFP levels were detected in OSN axon termini). The dye was electroporated by applying voltage pulses $(30 \mathrm{~V})$ until it became visible in distal neural processes of the PN, and left to diffuse for $60 \mathrm{~min}$. Brains were subsequently imaged by two-photon microscopy, as described above. 
To label single PNs, the DM2 glomerulus was first subjected to one cycle of exposure to 710 $\mathrm{nm}$ laser light to identify the cell bodies of DM2 PNs. Subsequently, the filled glass electrode was placed in the centre of the soma of one DM2 PN and the dye was electroporated by applying voltage pulses $(30 \mathrm{~V})$ until it became visible in distal neural processes of the neuron. The dye was left to diffuse for $60 \mathrm{~min}$, before the brains were fixed with $2 \%$ paraformaldehyde for $45 \mathrm{~min}$ and subjected to immunofluorescence using nc82 (1:20) and a-mouse Alexa488 (1:500), as described above. Images were acquired on a Zeiss LSM 880 Airy scan confocal microscope using a 40× objective (Plan Neofluar 40× oil immersion DIC objective; $1.3 \mathrm{NA}$ ).

\section{Molecular evolution and polymorphism analyses}

The Or $22 a / b$ gene tree was inferred using annotations after manual verification by BLASTing OR22a/b protein sequences (using tblastn with default settings, BLAST+ v2.7.1 ${ }^{72}$ ) to the genomes of $D$. simulans (r2.01), D. mauritiana (r1), D. yakuba (r1.04), D. sechellia (r1.3) and $D$. erecta (r1.3). Genomic regions were annotated using Wise2 $(\mathrm{v} 2.4 .1)^{73}$, with $D$. melanogaster's protein sequences as guides. All genes appeared intact and consistent with existing models, with one exception: $D$. mauritiana Or $22 b$ is truncated by $\sim 240$ bp due to a gap in the reference genome. The cDNAs were frame-aligned using Translator $\mathrm{X}^{74}$. The gene tree based on these alignments was inferred using MrBayes $(\mathrm{v} 3.2 .6)^{75}$ with the following settings: rate variation $=$ invariable+gamma, number of substitutions $=6$, substitution model $=$ default, number of generations $=10$, sample frequency $=10$, burn-in $=250$.

The Or22a/b topology outputted from MrBayes was used for PAML (v4.8) ${ }^{76}$ CodeML branch tests of protein evolution rate change. Seven models were tested, one with a freely varying $\omega$ value and six other models with 1, 4, 5, 7, 8 and $9 \omega$ values (CodeML parameters were all set to zero except for the following: seqtype $=1$, CodonFreq $=2$, ndata $=1$, model $=$ 2 , kappa $=2$, omega $=0.4$, fix_alpha $=1, \mathrm{ncatG}=5$, getSE $=1$, Small_Diff $=5 \mathrm{e}-7$, cleandata $=1$ ). Nested comparisons between these models using likelihood ratio tests identified a model with $5 \omega$ values to provide the best fit (Supplementary Data Table 8, Extended Data Fig. 11d).

Polymorphism data for $O r 22 a / b$ was extracted from previously published data. The $D$. simulans and $D$. sechellia datasets were from ${ }^{7,77}$ and the $D$. melanogaster datasets from ${ }^{78,79}$. VCFtools (v0.1.17) ${ }^{80}$ was used to extract variable sites from the respective VCF files, as well as to output allele frequencies. Variant calling in ${ }^{7}$ was based on a subversion of the $D$. simulans $\mathrm{r} 2$ genome, which is slightly different than $\mathrm{r} 2$ on FlyBase. This subversion is available at https://github.com/kern-lab/FILET/blob/master/simSechResults/dsimV2Mar2012_chrsonly.fa.bz2.

\section{Statistics and reproducibility}

Data were analysed and plotted using Excel and R (v3.2.3; R Foundation for Statistical Computing, Vienna, Austria, 2005; R-project-org).

\section{Extended Data}



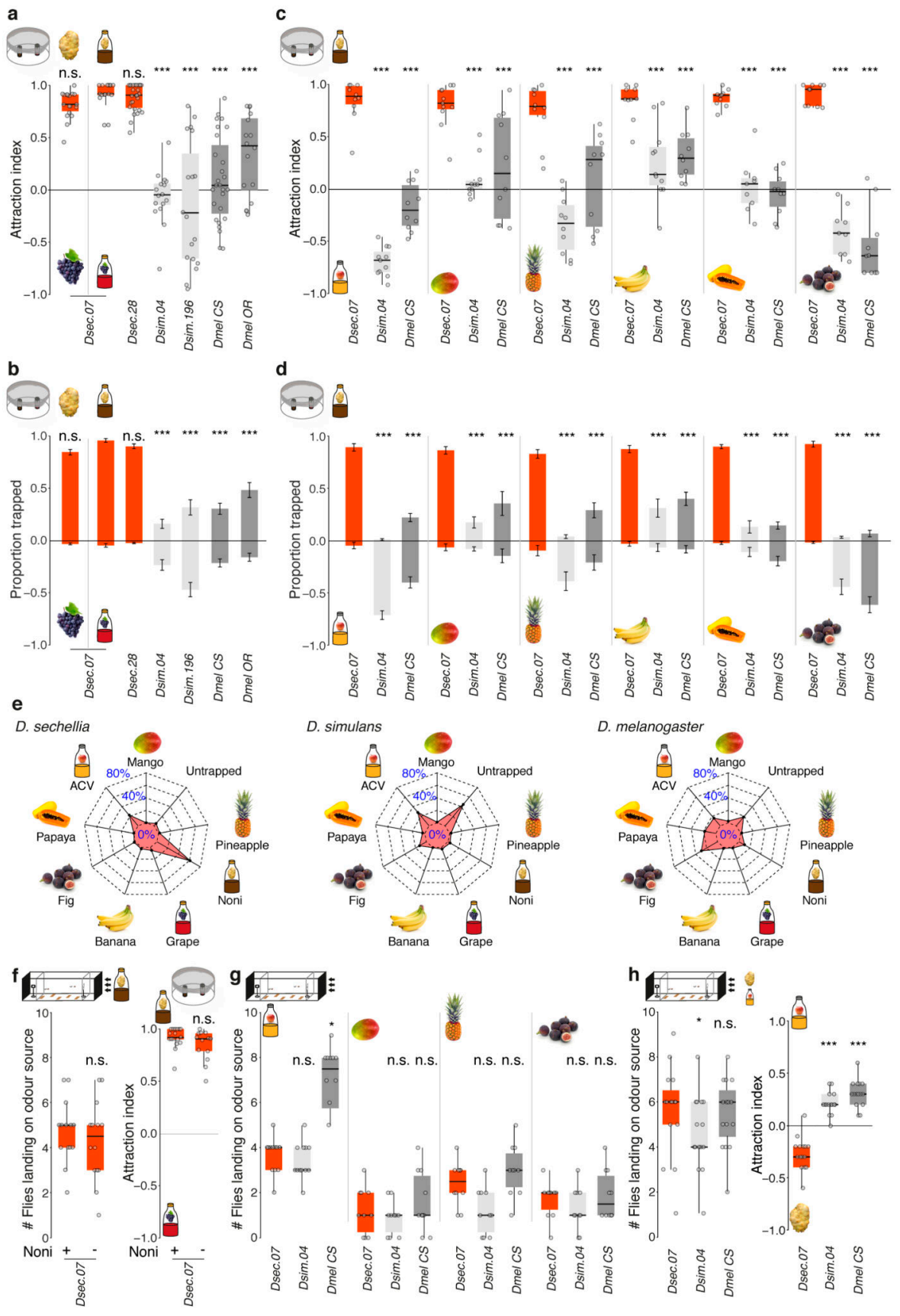

Extended Data Fig. 1. Species-specific short- and long-range behavioural responses to diverse fruit stimuli.

a, Data reproduced from Fig. 1c. Behavioural responses in a trap assay testing preferences between noni and grape, or between noni juice and grape juice $(n=15-27$ experiments; 22-25 females/experiment). Comparisons to Dsec.07 responses to noni juice are shown (a-d and $\mathbf{h}$ (right): pairwise Wilcoxon rank-sum test and $P$ values adjusted for multiple comparisons using the B\&H method): *** $P<0.001 ;$ n.s. $P>0.05$. 
$\mathbf{b}$, Proportion of flies (mean \pm SEM) in each stimulus trap for the assays shown in $\mathbf{a}$. Comparisons to Dsec.07 responses are shown.

c, Behavioural responses in a trap assay testing preferences between noni juice and diverse fruit juices or fruits for D. sechellia (DSSC 14021-0248.07), D. simulans (DSSC 14021-0251.004) and D. melanogaster (Canton-S) ( $\mathrm{n}=10-11$ experiments; $22-25$ females/ experiment). Comparisons to Dsec.07 responses are shown.

d, Proportion of flies (mean \pm SEM) in each stimulus trap for the assays shown in $\mathbf{c}$. Comparisons to Dsec.07 responses are shown.

e, Radar plot showing the mean percentage of flies per trap in a multiple choice trap assay with eight different stimuli for D. sechellia (DSSC 14021-0248.07), D. simulans (DSSC 14021-0251.004) and D. melanogaster (Canton-S) ( $\mathrm{n}=11$ experiments; $22-25$ females/ experiment). ACV $=$ Apple cider vinegar.

f, Left: Behavioural responses to noni juice in a wind tunnel assay of D. sechellia (DSSC 14021-0248.07) reared on standard food with (+) and without (-) noni supplement (KruskalWallis test: n.s. $P>0.05)$. Right: Behavioural responses in a trap assay testing preferences between noni juice and grape juice for D. sechellia (DSSC 14021-0248.07) reared on standard food with (+) and without (-) noni supplement (pairwise Wilcoxon rank-sum test: n.s. $P>0.05)$.

g, Behavioural responses to apple cider vinegar, mango juice, pineapple or fig in a wind tunnel assay of $D$. sechellia (DSSC 14021-0248.07), D. simulans (DSSC 14021-0251.004) and D. melanogaster (Canton-S) ( $\mathrm{n}=10-12$ experiments; 10 females/experiment).

Comparisons to Dsec.07 responses are shown (g and $\mathbf{h}$ (left): Kruskal-Wallis test with Dunn's post-hoc correction): * $P<0.05$; n.s. $P>0.05$.

$\mathbf{h}$, Behavioural responses in a wind tunnel assay testing preference between noni fruit and apple cider vinegar of D. sechellia (DSSC 14021-0248.07), D. simulans (DSSC 14021-0251.004) and D. melanogaster (Canton-S) ( $\mathrm{n}=15$ experiments; 10 females/ experiment). Left: total number of flies landing on an odour source. Comparisons to Dsec.07 responses are shown. Right: attraction index calculated as: (flies landing on apple cider vinegar - flies landing on noni)/flies landing on either source. Comparisons to Dsec.07 responses are shown. 


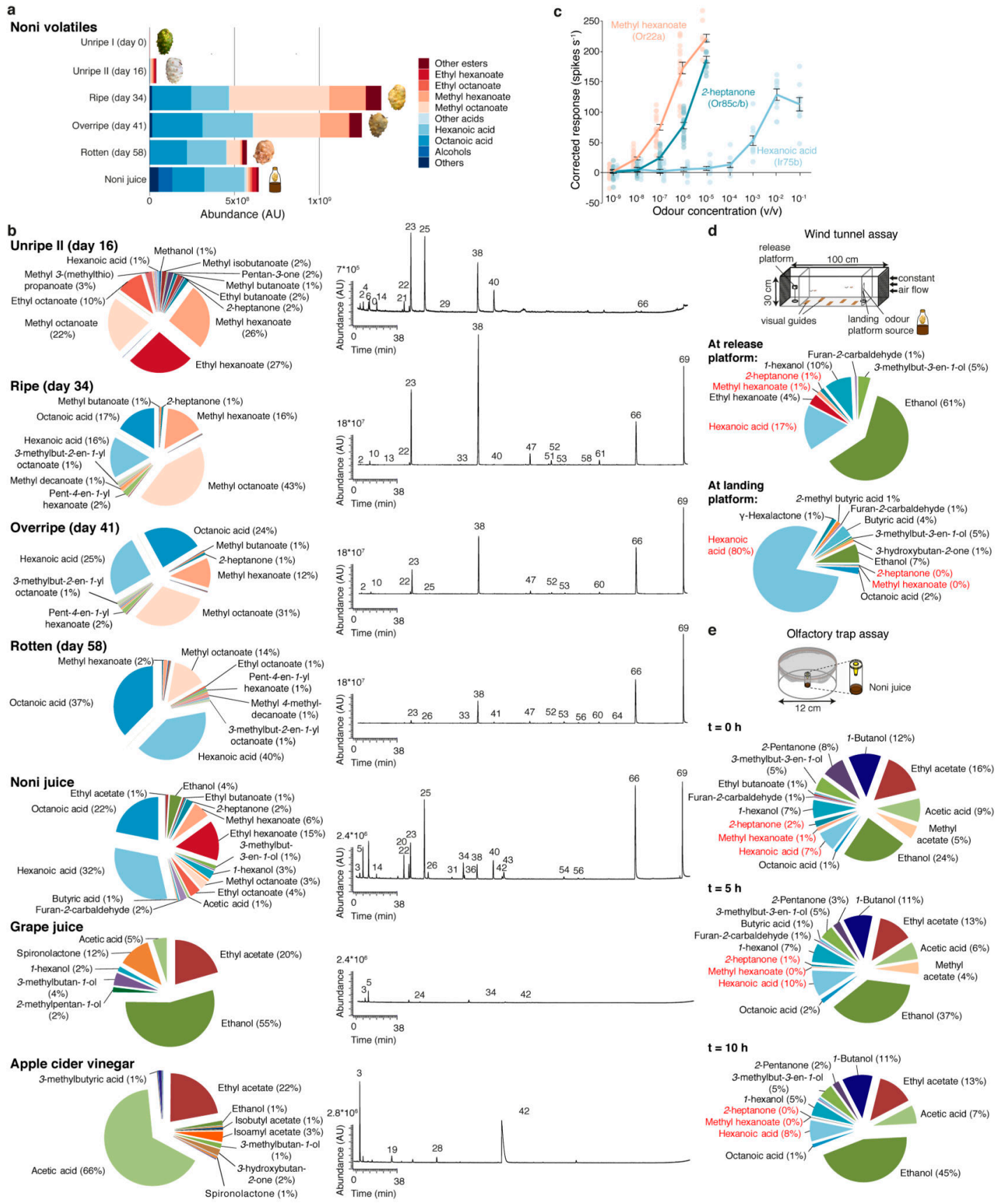

Extended Data Fig. 2. Chemicals emitted by natural odour sources and odour bouquet changes in behavioural assays.

a, Principal constituents of the odour bouquet of noni fruit at different ripening stages and commercial noni juice, as determined by gas chromatography/mass spectrometry. $\mathrm{AU}=$ arbitrary units.

b, Chemical composition of the odour bouquet of noni fruit at different stages of ripening, noni juice, grape juice and apple cider vinegar. Representative gas chromatograms are shown 
on the right. Numbers correspond to compounds as listed in Supplementary Table 1 (not all identified peaks are shown).

c, Dose-dependent electrophysiological responses of Or22a, Or85c/b and Ir75b neurons in wild-type D. sechellia (DSSC 14021-0248.07) to their best odour agonists (mean \pm SEM and individual data points, $\mathrm{n}=7-20$, females). The contribution of Or35a neurons (whose spiking is difficult to separate from Ir75b neurons in ac3I) to hexanoic acid responses is likely to be minimal (Fig. 2b). D. sechellia Or22a and Or85c/b dose response data are replotted from Fig. 3a.

d, Chemical profile of odours collected by SPME at the release and landing platforms in the wind tunnel assay within the first 10 min after noni juice application.

e, Chemical profile of odours collected by SPME in the trap assay arena within 5 min after placement of a trap (i.e., $\mathrm{t}=0 \mathrm{~h}$ ), and after $5 \mathrm{~h}$ and $10 \mathrm{~h}$, using noni juice as stimulus. " $0 \%$ " indicates only trace proportions of the compound were detected. 


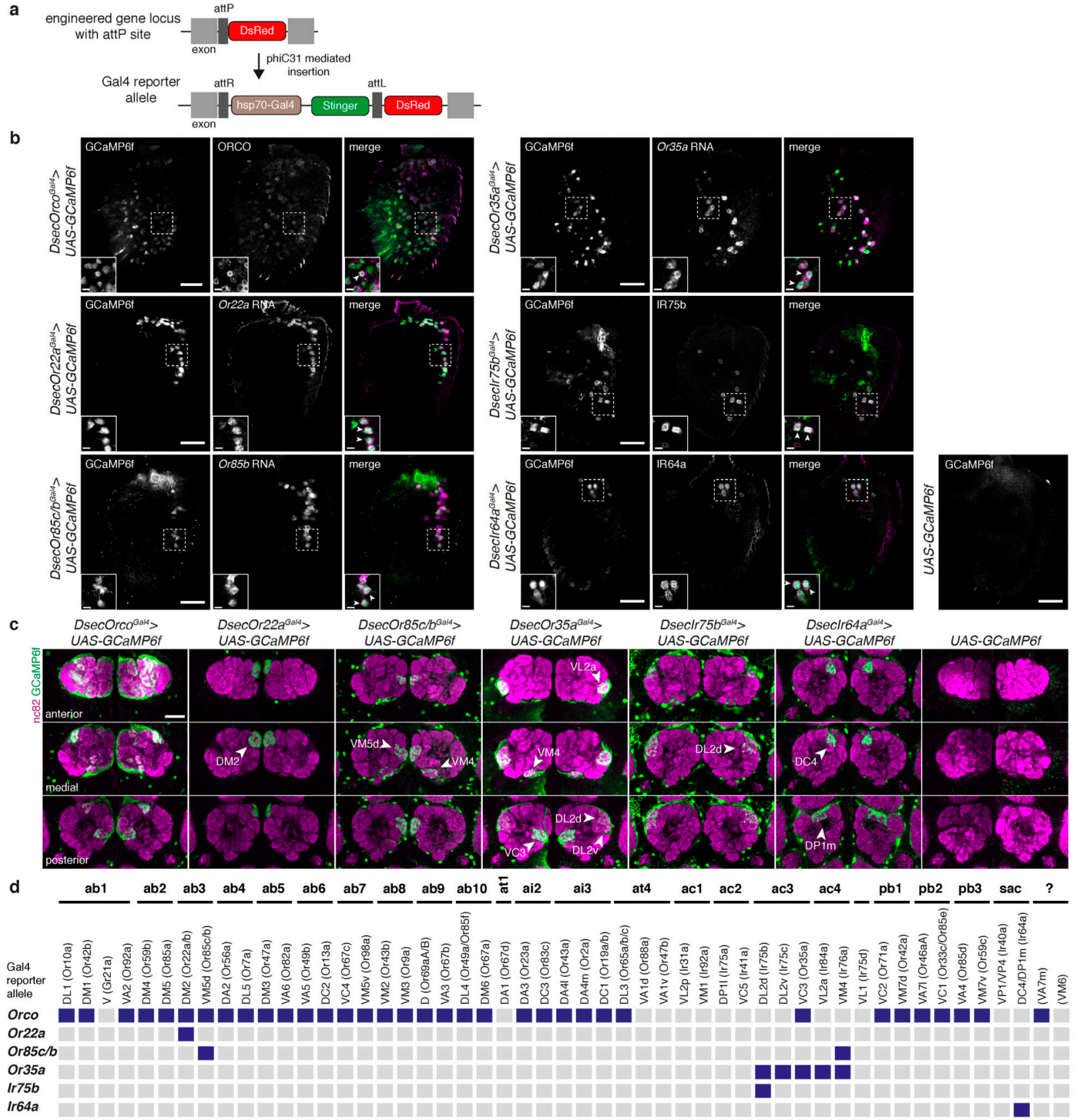

Extended Data Fig. 3. Olfactory sensory neuron Gal4 driver lines in D. sechellia.

a, Schematic of the Gal4 reporter allele generation strategy, through CRISPR/Cas9-mediated integration of an attP site (marked by $3 x P 3: D s R e d$ ) into the desired Or or Ir gene (see Extended Data Fig. 5-6 for details on specific alleles), followed by introduction of a Gal4 open reading frame via phiC31-mediated transgenesis.

b, Co-expression of the indicated $\mathrm{Or}^{\mathrm{Gal4}}$-driven, $\mathrm{Ir}^{\mathrm{Gal4}}$-driven or control background GCaMP6f signal (detected by a-GFP) with the corresponding receptor protein or RNA in whole-mount antennae. Arrowheads point to examples of co-labelled cells. Scale bars, 25 
$\mu \mathrm{m}$; inset scale bars, $5 \mu \mathrm{m}$. While Or $22 a^{G a l 4}$ and Ir64aGal4 completely recapitulate endogenous receptor expression, $\mathrm{OrCo}^{\mathrm{Gal}} 4$ and $\mathrm{Or} 85 \mathrm{c} / \mathrm{b}^{\mathrm{Gal4}}$ lack expression in some receptor-expressing neurons. Or $35 a^{\text {Gal4 }}$ and $\mathrm{Ir} 75 b^{\mathrm{Gal} 4}$ might be expressed in ectopic cells (see also c-d) or the protein/RNA signal for these receptor genes could be below the detection threshold.

c, Expression of the indicated $\mathrm{Or}_{r}{ }^{\text {Gal4}}$-driven, ${ }_{I}{ }^{G a l 4}$-driven or control background GCaMP6f signal (detected by a-GFP) in glomeruli of whole-mount antennal lobes. Three focal planes of the neuropil (visualised with nc82 (magenta)) are shown. Images were registered to a $D$. sechellia reference brain (see Methods) for better comparison of antennal lobe structure. Scale bar, $25 \mu \mathrm{m}$.

d, Summary of the glomerular labelling by $\mathrm{Or}_{r} \mathrm{Gal4}_{4}$ or $\mathrm{II}^{\mathrm{Gal} 4}$ drivers as characterised in c (dark blue indicates GCaMP6f signal was detected in at least 3/3 independent brains).

Glomeruli are organised by the compartmentalisation of the corresponding OSN populations into different sensilla classes (based on data in D. melanogaster ${ }^{81}$, ab: antennal basiconic; at: antennal trichoid; ai; antennal intermediate; ac: antennal coeloconic, $\mathrm{pb}=$ palp basiconic, $\mathrm{sac}$ = sacculus, ? = OSN population unknown). Orco ${ }^{\text {Gal4 }}$ is expressed in most but not all (e.g., Or67d/DA1) expected OSN populations; Or35a Gal4 and $O r 85 c / b^{G a l 4}$ display some ectopic expression as inferred by their labelling of more than one glomerulus. 


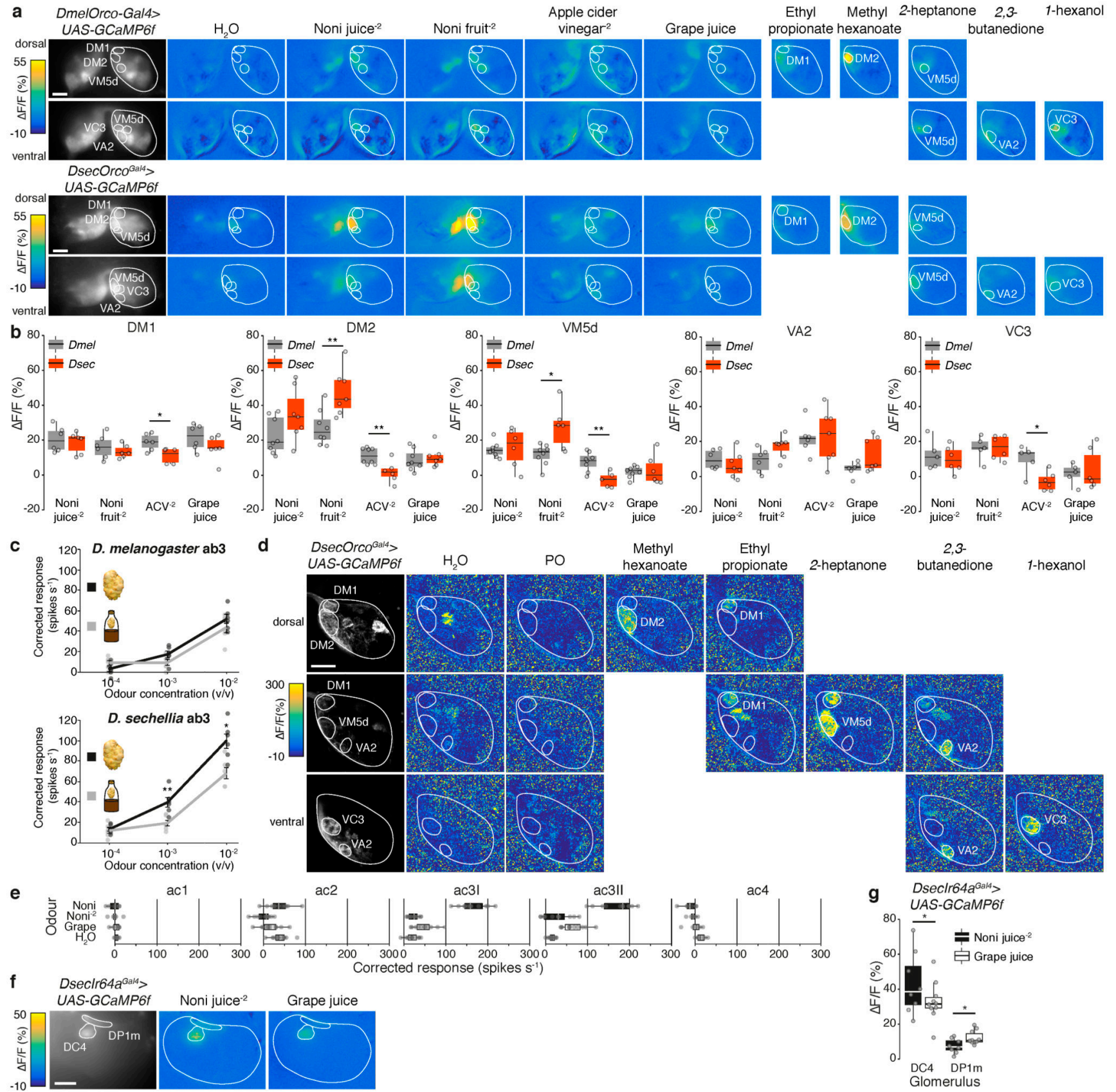

Extended Data Fig. 4. Comparative olfactory representations of noni in D. melanogaster and $D$. sechellia.

a, Representative odour-evoked calcium responses in the axon termini of Orco OSNs in the antennal lobes of D. melanogaster (Orco-Gal4/Orco-Gal4;UAS-GCaMP6f/UAS-GCaMP6f) and D. sechellia (UAS-GCaMP6f/UAS-GCaMP6f;;DsecOrcoGal4/+;), acquired by widefield imaging. Left images: raw fluorescence signals. Right images: relative increase in GCaMP6f fluorescence $(\Delta \mathrm{F} / \mathrm{F} \%)$ after stimulation with the indicated complex stimuli and single odours. Diagnostic odours: methyl hexanoate $\left(10^{-6}(\mathrm{v} / \mathrm{v})\right)$ for Or22a/(b)/DM2, ethyl propionate $\left(10^{-4}\right)$ for Or42b/DM1, 2-heptanone $\left(10^{-5}\right)$ for Or85c/b/VM5d, 2,3-butanedione 
$\left(10^{-4}\right)$ for Or92a/VA2 and 1-hexanol $\left(10^{-4}\right)$ for Or35a/VC3. Glomerular boundaries, and the entire antennal lobe, are outlined. Scale bars, $50 \mu \mathrm{m}$.

$\mathbf{b}$, Quantification of odour-evoked calcium responses for the animals represented in $\mathbf{a}$.

Maximum calcium response amplitudes for each experiment are plotted ( $\mathrm{n}=5-8$ females).

Significantly different responses of species to the same stimulus are shown (Wilcoxon signed-rank test): ** $P<0.01 ; * P<0.05$.

c, Combined electrophysiological responses of neurons in the ab3 sensillum in $D$.

melanogaster (top) and D. sechellia (bottom) upon stimulation with increasing

concentrations of noni juice or noni fruit extract (mean \pm SEM and individual data points, $n$ $=6$, females). Significant differences in responses are shown (pairwise Wilcoxon rank-sum test): ** $P<0.01 ; * P<0.05$. Note that responses of $D$. sechellia are stronger to noni fruit than noni juice, which may reflect the lower abundance of relevant ligands in the juice.

d, Representative odour-evoked calcium responses in the axon termini of Orco OSNs in the antennal lobe of D. sechellia (UAS-GCaMP6f/UAS-GCaMP6f;;DsecOrco ${ }^{\text {Gal4/+t) acquired }}$ by two-photon imaging. Three focal planes are shown, revealing different glomeruli along the dorsoventral axis. Left column: raw fluorescence images. Other columns: relative increase in GCaMP6f fluorescence $(\Delta \mathrm{F} / \mathrm{F} \%)$ after stimulation with diagnostic odours. Scale bar, $25 \mu \mathrm{m}$.

e, Electrophysiological responses in the antennal coeloconic (ac) sensilla classes to the indicated stimuli ( $\mathrm{n}=6-11$ sensilla, females) in D. sechellia (DSSC 14021-0248.07)

representing the summed, solvent-corrected activities of the two or three neurons they house. f, Representative odour-evoked calcium responses in the axon termini of Ir64a OSNs in the D. sechellia antennal lobe (genotype: UAS-GCaMP6f/UAS-GCaMP6f;;DsecIr64aGal4/+) acquired by widefield imaging. Left images: raw fluorescence signals. Right images: relative increase in GCaMP6f fluorescence $(\Delta \mathrm{F} / \mathrm{F} \%)$ after stimulation with noni juice $\left(10^{-2}\right)$ or grape juice. Scale bar, $25 \mu \mathrm{m}$.

g, Quantification of odour-evoked calcium responses for the animals represented in $\mathbf{f}$.

Maximum calcium response amplitudes for each experiment are plotted $(n=7-10$, females $)$. Comparisons of responses to noni $\left(10^{-2}\right)$ and grape juice are shown (Wilcoxon signed-rank test): $* P<0.05$. 

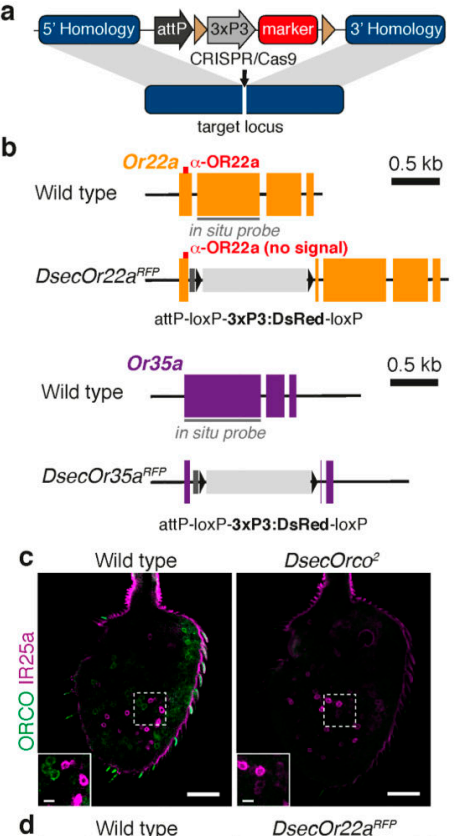

DsecOrco ${ }^{2}$
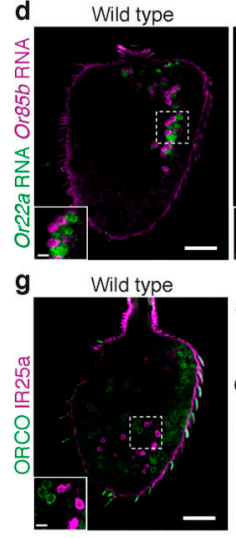

Dsecorco ${ }^{+}$

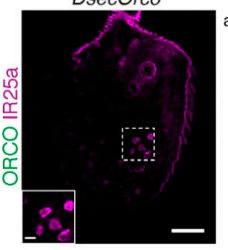

h

DsecOr22a ${ }^{\text {RFP }}$

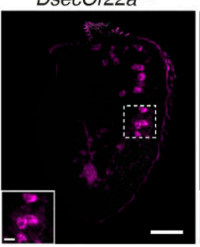

ab3 ${ }^{\text {a }}$ Methylhex

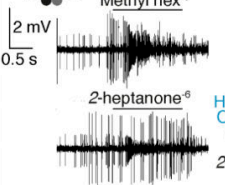

ab3 Methylhex

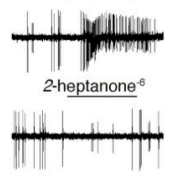

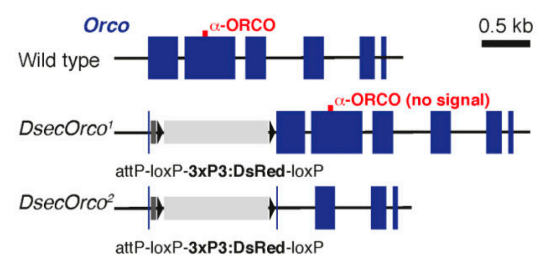

Or85c
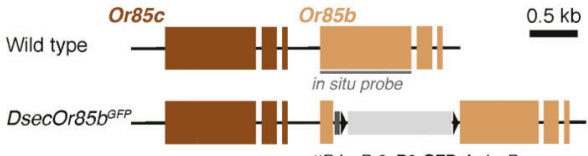

attP-loxP-3xP3:GFPnls-loxP

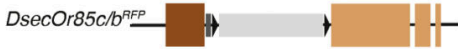

attP-loxP-3xP3:DsRed-loxP

e Wild type DsecOr35a ${ }^{\text {RFP }}$

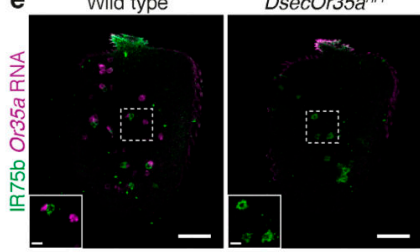

DsecOr85b ${ }^{G F P}$

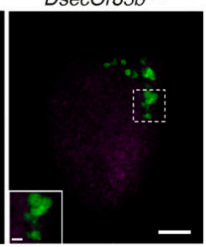

f Wild type

DsecOr22a ${ }^{\text {RFP }}$

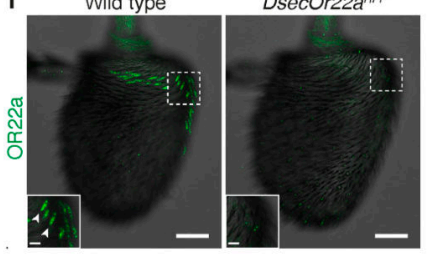

Odour

Methylar ab3A activity
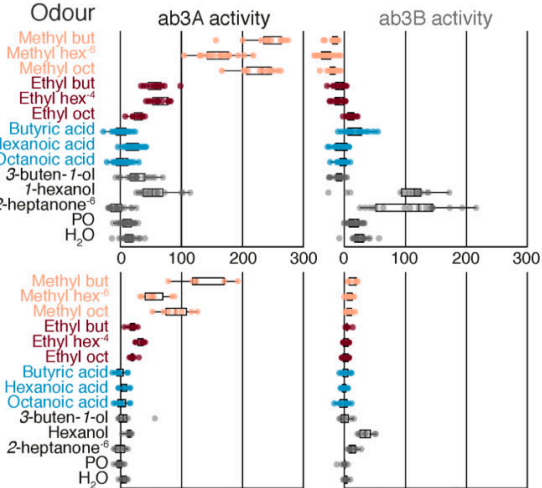

$+\square^{-1}$

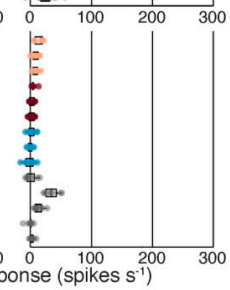

in-frame alternative start codons

Extended Data Fig. 5. Generation and validation of loss-of-function alleles of $\mathrm{D}$. sechellia $\mathrm{Or}$ genes.

a, Schematic of the strategy for generating olfactory receptor mutant alleles, through integration of an eye-expressed fluorescent marker (3xP3:DsRed or 3xP3:GFPnls) into the desired locus via CRISPR/Cas9-cleavage induced homologous recombination. Brown triangles: $l_{x} P$ sites for removal of the fluorescent marker via Cre recombination.

b, Schematics depicting Or gene organisation, the structure of mutant alleles, and the location of the sequences encoding antibody epitopes. For $D_{s e c O r c o}{ }^{l}$ the fluorescent marker 
was integrated into the first coding exon; for $\mathrm{DsecOrco}^{2}$ the marker replaces parts of exons 1 and 3 and the whole of exon 2. DsecOr22a ${ }^{R F P}$ carries the fluorescent marker in the first coding exon close to the start codon. DsecOr $35 a^{R F P}$ lacks most of exons 1 and 2 . For $D s e c O r 85 b^{G F P}$ the marker was integrated into exon 1 ; for $D s e c O r 85 c / b^{R F P}$ the marker replaces most of the $\mathrm{Or} 85 \mathrm{c}$ gene and part of exon 1 of $\mathrm{Or} 85 \mathrm{~b}$.

c, Immunofluorescence for ORCO and IR25a (as internal staining control) on whole-mount antennae from wild-type and $\mathrm{DsecOrco}^{2}$ animals. c-g: Scale bars, $25 \mu \mathrm{m}$; inset scale bars, 5 $\mu \mathrm{m}$.

d, RNA FISH for Or22a and Or85b on whole-mount antennae from wild-type, DsecOr $22 a^{R F P}$ and DsecOr85b ${ }^{G F P}$ mutant animals.

e, Immunofluorescence for IR75b and RNA FISH for Or35a on whole-mount antennae from wild-type and DsecOr35a ${ }^{R F P}$ mutant animals. Arrowheads indicate Or35a-expressing cells. Note that Or35a neurons also pair with Ir75c neurons in ac3II sensilla ${ }^{15}$, which is reflected in Or35a-positive cells that are not paired with IR75b-expressing cells in wild-type antennae. f, Immunofluorescence for OR22a on whole-mount antennae from wild-type and DsecOr22a ${ }^{R F P}$ mutant animals. Arrowheads indicate sensilla housing Or22a neurons.

g, Left panel: immunofluorescence for ORCO and IR25a (as internal staining control) on whole-mount antennae from wild-type (same picture as shown in c) and $\mathrm{DsecOrco}^{1}$ animals. Central panel: electrophysiological responses in the two neurons of the ab3 sensillum (see Fig. 2a) to odours present in noni in D. sechellia (DSSC 14021-0248.07) and DsecOrco ${ }^{1}$ mutants $\left(n=5-20\right.$, females). Representative response traces to methyl hexanoate $\left(10^{-6}\right)$ and 2-heptanone $\left(10^{-6}\right)$ are shown to the left. Data points represent the solvent-corrected activities per neuron. D. sechellia wild-type responses are replotted from Fig. 2a.

Surprisingly, even though ORCO expression is undetectable by immunofluorescence, weak electrophysiological responses in ab3 sensilla (and other ORCO-dependent sensilla (data not shown)) can be detected. These observations suggest that trace levels of functional ORCO are produced from this allele, potentially through use of in-frame start codons downstream of the marker insertion site (see $\mathbf{h}$ ).

h, A schematic depicting the location of the Orco start codon, the fluorescent marker insertion site of the DsecOrco $^{I}$ allele and downstream potential alternative in-frame start codons. 
a

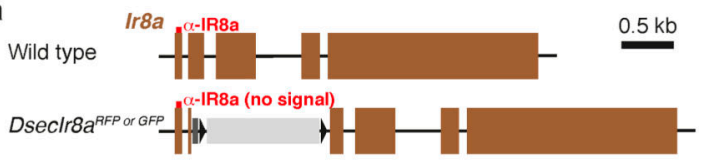

attP-loxP-3xP3:DsRed-loxP or attP-loxP-3xP3:GFPnls-loxP

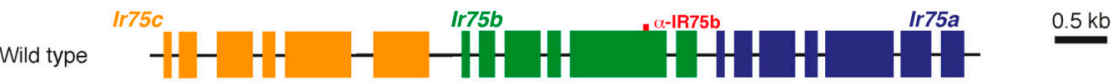

Dseclr75b $b^{\text {RFP }}$

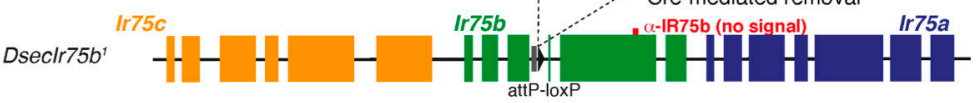

Dseclr75b Ir75c

Dseclr75b ${ }^{2}$
C

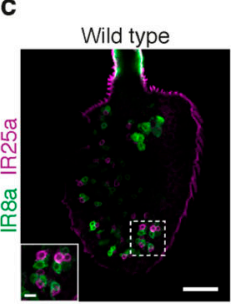

Dseclr8a ${ }^{G F P}$

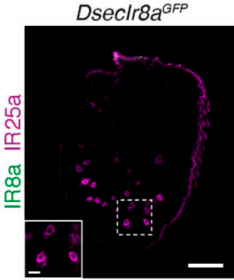

Dsecir $75 b^{2}$

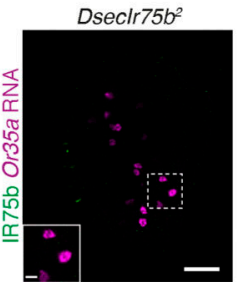

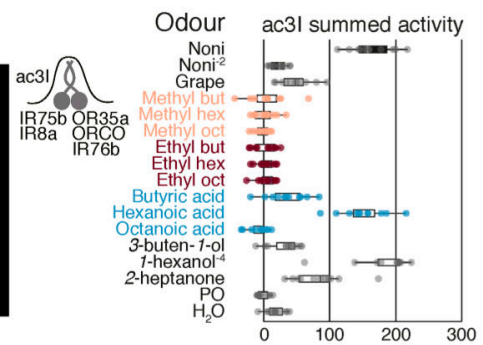

$\operatorname{ac31} 2$

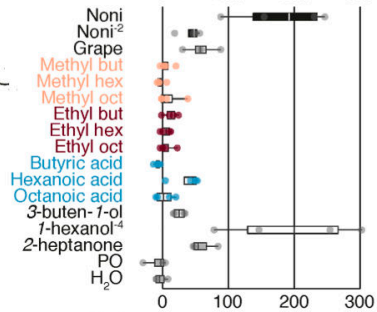

ac31/2

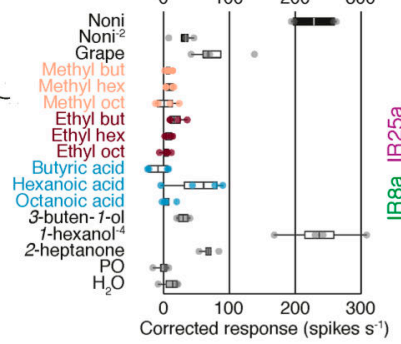

d

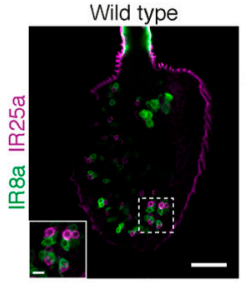

Dseclr8a ${ }^{R F P}$

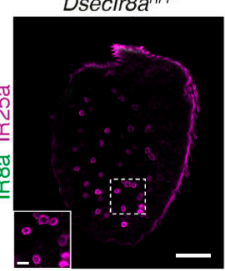

Dseclr8a ${ }^{G F P}$

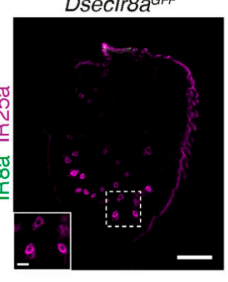

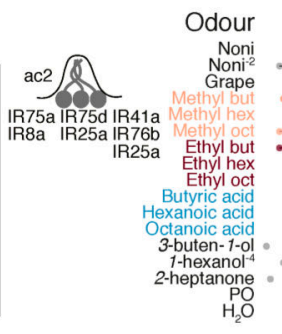

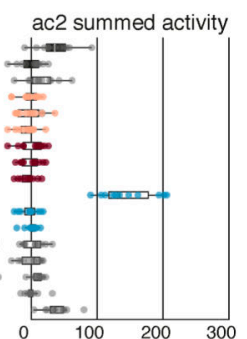

$\sqrt[a c 2]{x-1}$

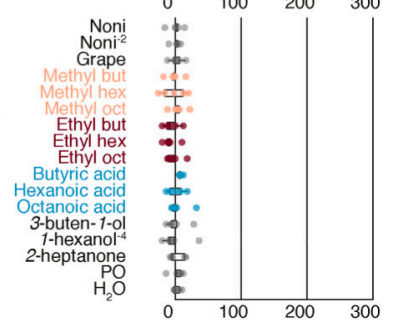

ac2

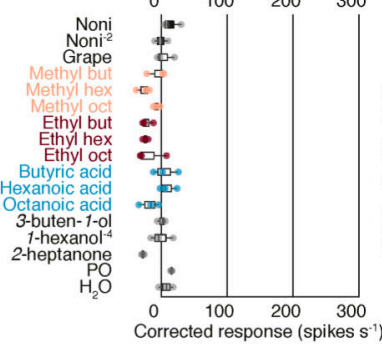

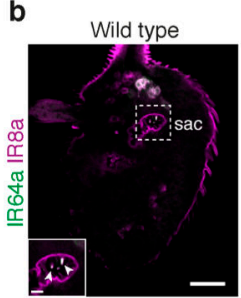

Dseclr64a ${ }^{\text {RFP }}$

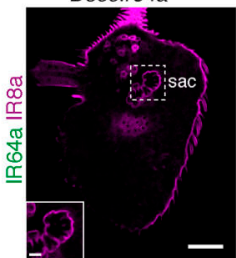

\section{e}

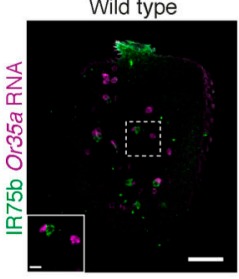

Dseclr $75 b^{1}$

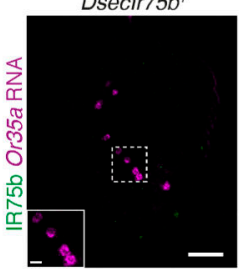

Dseclr $75 b^{2}$

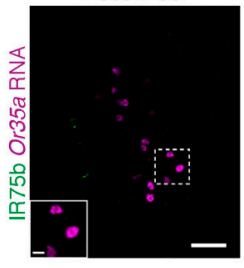

Extended Data Fig. 6. Generation and validation of loss-of-function alleles of D. sechellia Ir genes.

a, Schematics depicting Ir gene organisation, the structure of mutant alleles, and the sequences encoding antibody epitopes. For $D s e c I r 8 a^{R F P / G F P}$ the fluorescent marker was integrated into the first coding exon. For DsecIr $64 a^{R F P}$ the marker replaces parts of exon 2. $D$ secIr $75 b^{R F P}$ lacks parts of exons 3 and 4, while for DsecIr $75 b^{G F P}$ the marker was integrated into exon 3 . For both alleles of $\operatorname{Ir} 75 b$, the fluorophore was removed via Cremediated recombination to produce $\operatorname{Ir} 75 b^{1}$ and $\operatorname{Ir} 75 b^{2}$. 
b, Immunofluorescence for IR64a and IR8a (as internal staining control) on whole-mount antennae from wild-type and DsecIr64a ${ }^{R F P}$ mutant animals. Arrowheads indicate the Ir64a neuron dendrites innervating sensilla in the sacculus (sac). Scale bars, $25 \mu \mathrm{m}$; inset scale bars, $5 \mu \mathrm{m}$.

c, Left panel (top): immunofluorescence for IR8a and IR25a (as internal staining control) on whole-mount antennae from wild-type and DsecIr $8 a$ GFP animals. Left panel (bottom): immunofluorescence for IR75b and RNA FISH for Or35a on whole-mount antennae from DsecIr $75 b^{2}$ mutant animals. Scale bars, $25 \mu \mathrm{m}$; inset scale bars, $5 \mu \mathrm{m}$. Right panel: electrophysiological responses in the ac3I sensillum (neurons housed are indicated in the cartoon) to noni juice, grape juice and odours present in noni ( $\mathrm{n}=4-11$, females) in $D$. sechellia (DSSC 14021-0248.07) and olfactory receptor mutants affecting the Ir75b neuron $\left(D s e c I r 8 a^{G F P}, D s e c I r 75 b^{2}\right.$ ). Data points represent the summed, solvent-corrected activities of the sensillum. D. sechellia wild-type responses are replotted from Fig. 2b. Note that the Or35a neuron exhibits residual responses to hexanoic acid in the DsecIr $8 a$ and DsecIr $75 b$ olfactory receptor mutants (see also Fig. 2b).

d, Left panel: immunofluorescence for IR8a and IR25a (as internal staining control) on whole-mount antennae from wild-type (same picture as shown in c) and DsecIr $8 a^{R F P}$ and ${ }_{\text {SsecIr } 8 a}{ }^{G F P}$ (same picture as shown in c) animals. Scale bars, $25 \mu \mathrm{m}$; inset scale bars, $5 \mu \mathrm{m}$. Right panel: electrophysiological responses in the ac2 sensillum (neurons housed are indicated in the cartoon) to noni juice, grape juice and odours present in noni ( $\mathrm{n}=3-11$, females) in D. sechellia (DSSC 14021-0248.07) and receptor mutants affecting the Ir75a neuron (DsecIr8a $\left.{ }^{R F P}, D s e c I r 8 a G F P\right)$. Data points represent the summed, solvent-corrected neuronal activities of the sensillum. $D$. sechellia wild-type responses to noni and grape juice are as shown in Extended Data Fig. 4e.

e, Immunofluorescence for IR75b and RNA FISH for Or35a on whole-mount antennae from wild-type and $D$ secIr $75 b^{1}$ and $D \operatorname{secIr} 75 b^{2}$ (same picture as shown in c) animals. Scale bars, $25 \mu \mathrm{m}$; inset scale bars, $5 \mu \mathrm{m}$. 

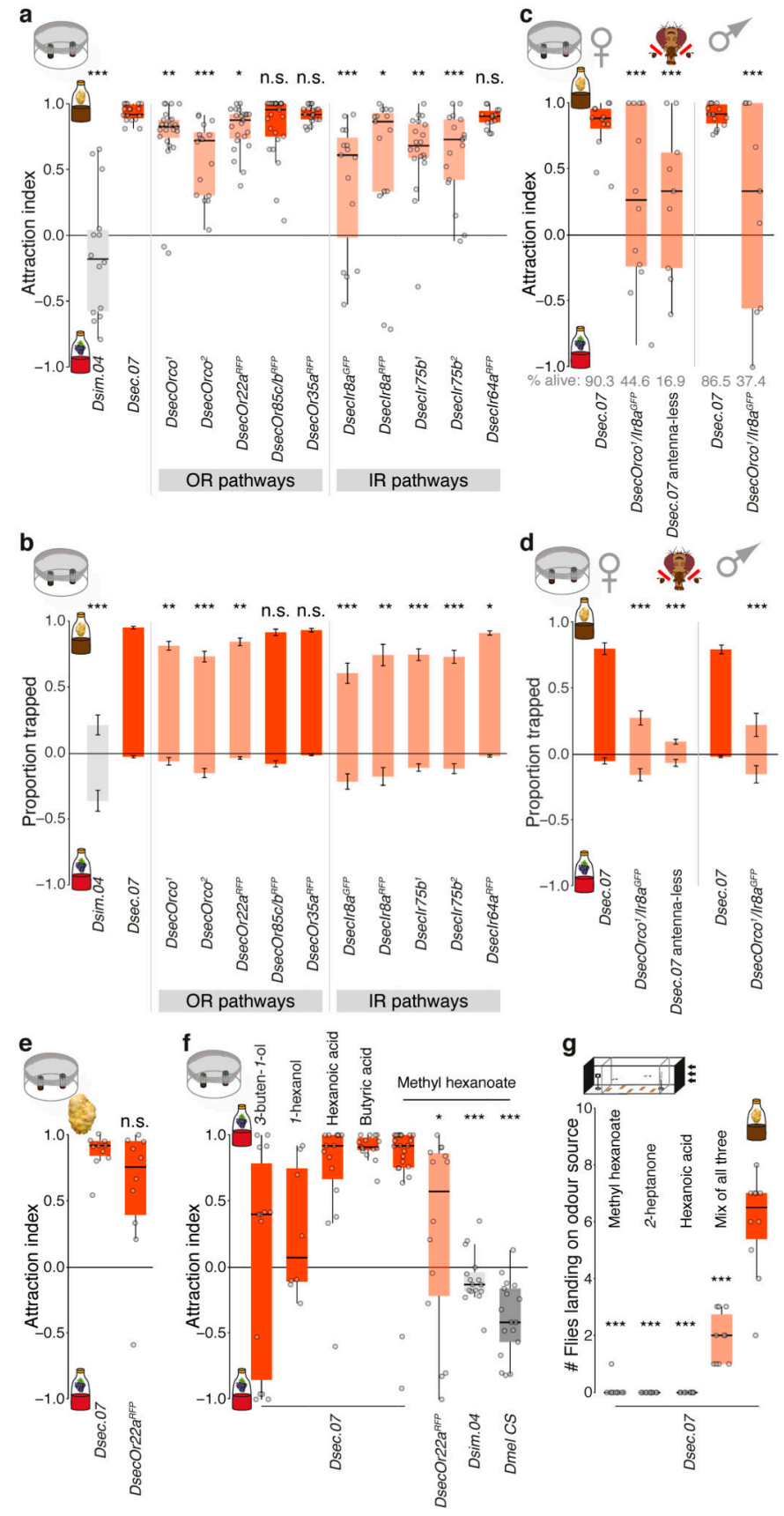

Extended Data Fig. 7. Genetic and chemical contributions promoting attraction of $D$. sechellia to noni.

a, Data reproduced from Fig. 2d. Behavioural responses in a trap assay testing preference of the indicated genotypes for noni juice or grape juice $(n=13-25$ experiments; $22-25$ females/ experiment). Comparisons to Dsec.07 responses are shown (a-d and f: pairwise Wilcoxon rank-sum test and $P$ values adjusted for multiple comparisons using the B\&H method): red bars, no significant difference; salmon bars, significantly different response; $* * * P<0.001$; ** $P<0.01$; * $P<0.05$; n.s. $P>0.05$. 
b, Proportion of flies (mean \pm SEM) in each stimulus trap for the assays shown in $\mathbf{a}$. Comparisons to Dsec.07 responses are shown.

c, Olfactory responses in a trap assay testing preferences between noni juice and grape juice of D. sechellia (DSSC 14021-0248.07), DsecOrco ${ }^{1} / \mathrm{Ir}_{\mathrm{r}} \mathrm{G}^{G F P}$ double mutants and D. sechellia (DSSC 14021-0248.07) whose third antennal segments were removed (antenna-less) ( $\mathrm{n}=$ 9-15 experiments; $22-25$ females or males, as indicated/experiment). These data represent the same experiments shown in Fig. 2e, but attraction indices were calculated here taking only alive flies into account. The percentages of flies alive at the end of the assay are indicated below, revealing the high mortality rate of antenna-less flies and $\mathrm{DsecOrco}^{1 /}$ Ir8a ${ }^{G F P}$ double mutants ( $\mathrm{DsecOrco}^{2} / \mathrm{Ir} 8 \mathrm{a}{ }^{G F P}$ mutants appeared to be non-viable). Normally, trap assay experiments with $>25 \%$ animal mortality were discarded; see Methods.

Comparisons to Dsec.07 responses are shown.

d, Proportion of flies (mean \pm SEM) in each stimulus trap for the assays shown in $\mathbf{c}$. Comparisons to Dsec.07 responses are shown.

e, Behavioural responses in a trap assay testing preferences between noni fruit and grape juice of Dsec.07 and DsecOr22a ${ }^{R F P}$ flies. Comparisons to Dsec.07 responses are shown (pairwise Wilcoxon rank-sum test): n.s. $P>0.05$.

f, Behavioural responses in a trap assay testing preferences of D. sechellia (DSSC 14021-0248.07) between grape juice and $10^{-2}$ dilutions of the indicated odours in grape juice of D. sechellia (DSSC 14021-0248.07), DsecOr22a ${ }^{R F P}$, D. simulans (DSSC 14021-0251.004) and D. melanogaster (Canton-S). Comparisons to Dsec.07 responses to methyl hexanoate are shown.

g, Behavioural responses in a wind tunnel assay testing attraction of D. sechellia (DSSC 14021-0248.07) to three single noni odours ( $10^{-2}$ in water), a mix of all three in the approximate proportions of ripe noni fruit (1:0.04:1, methyl hexanoate:2heptanone:hexanoic acid) and noni juice ( $\mathrm{n}=10$ experiments; 10 females/experiment). Comparisons to Dsec.07 responses to noni juice are shown (Kruskal-Wallis test with Dunn's post-hoc correction): $* * * P<0.001$. 


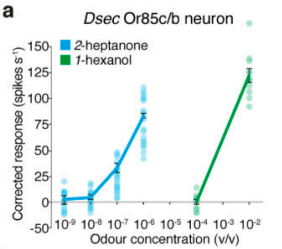

b D. sechellia

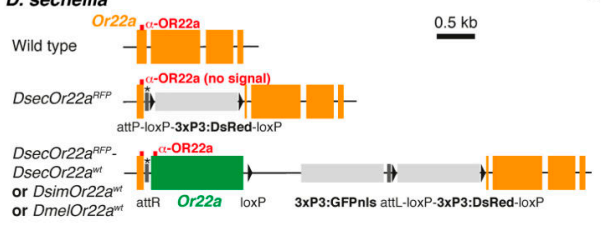

D. melanogaster

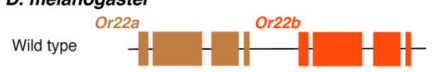

DmeiOr5sa/bif

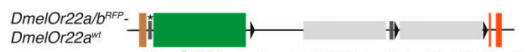

attR Or22a loxP $3 \times$ PP3:GFPnis attL-10xP-3xP3:DsRed-loxP
D. simulans

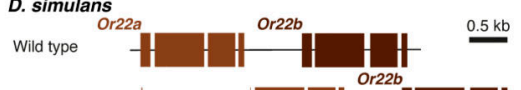

DsimOr22aAFP

DsimOr22a/b PFP $_{\text {attP-loxP-3XP3:DsRed-loxP }}$
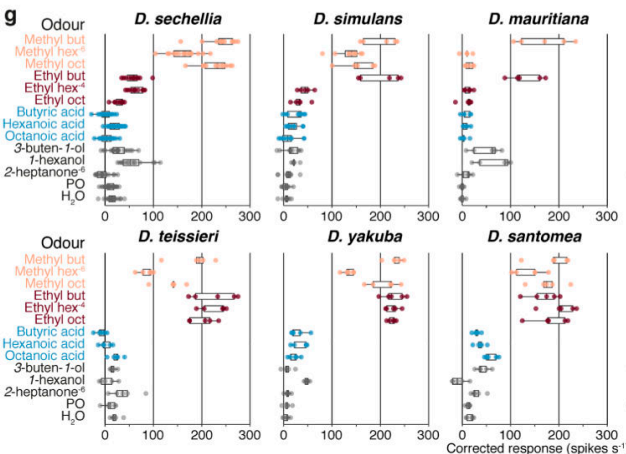

h Odour DsecOr22a $a^{\text {RFP }}$
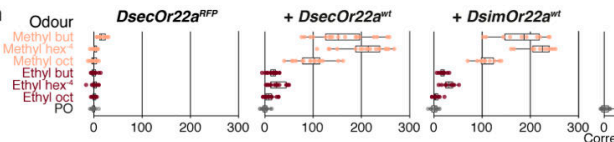

i Odour DmelOr22a/banselo
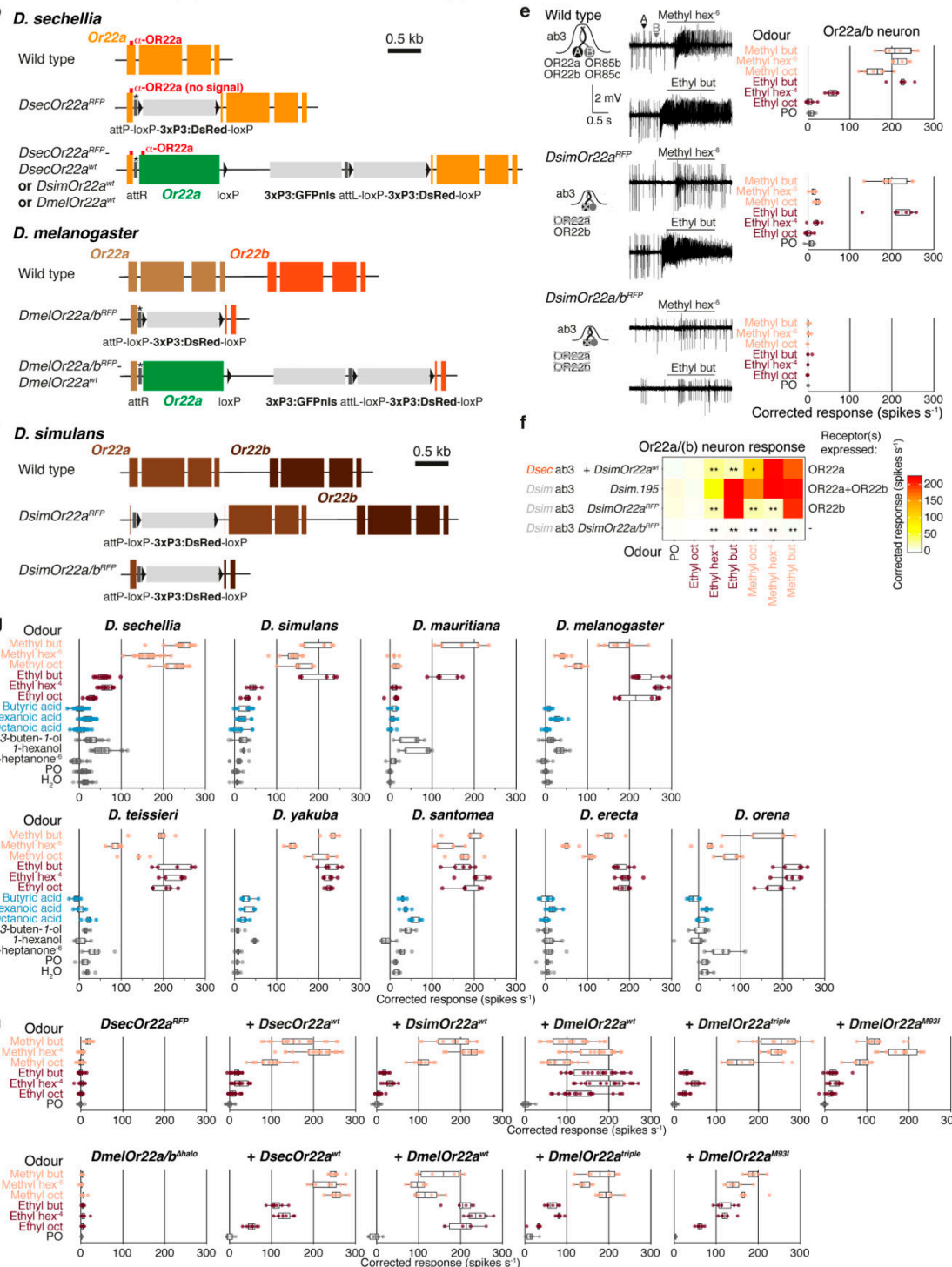

DsimOr22a $a^{\text {PFP }}$ Methyl he

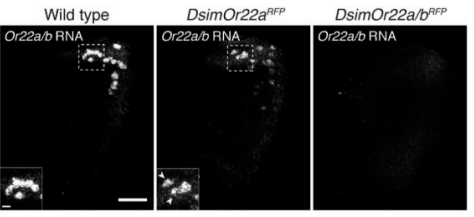

DmelOr22ant
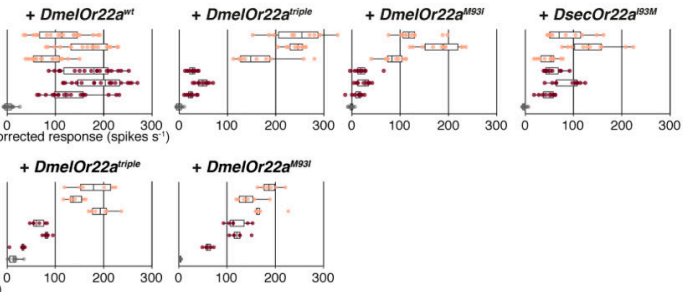

Extended Data Fig. 8. Odour-tuning properties of drosophilid Or85c/b and Or22a/(b) neurons and genomic modifications of the $\mathrm{Or} 22 \mathrm{a} /(\mathrm{b})$ loci.

a, Left: dose-dependent electrophysiological responses of Or85c/b neurons (ab3B) in wildtype D. sechellia (DSSC 14021-0248.07) to 2-heptanone and 1-hexanol (mean \pm SEM and individual data points; $\mathrm{n}=10-20$, females). Right: dose-dependent electrophysiological responses of Or22a neurons (ab3A) in wild-type D. sechellia (DSSC 14021-0248.07) to methyl butanoate, methyl hexanoate and methyl octanoate (mean \pm SEM and individual data points; $n=10-20$, females). The dose response curves for 2-heptanone and methyl hexanoate are replotted from Fig. $3 \mathrm{a}$.

Nature. Author manuscript; available in PMC 2020 September 04. 
b, Schematics depicting the arrangement of wild-type, mutant and rescue allele versions of DsecOr22a (top) and DmelOr22a/Or22b (bottom). Asterisk = stop codon preventing read through from the endogenous Or22a open reading frame.

c, Schematics depicting the arrangement of wild-type and mutant alleles of DsimOr22a/ Or22b.

d, RNA FISH for Or22a on whole-mount antennae from wild-type $D$. simulans (DSSC 14021-0251.195), DsimOr22a ${ }^{R F P}$ and DsimOr22a/b ${ }^{R F P}$ mutant animals. As Or22a shares $85 \%$ sequence similarity with $\operatorname{Or} 22 b$, the Or22a probe hybridises with transcripts from both genes. Arrowheads indicate Or22b-expressing cells in DsimOr22a ${ }^{R F P}$. Scale bar, $25 \mu \mathrm{m}$; inset scale bar, $5 \mu \mathrm{m}$.

e, Electrophysiological responses of Or22a/b neurons to different esters in wild-type $D$. simulans (DSSC 14021-0251.195) and receptor mutants (DsimOr22a ${ }^{R F P}$, DsimOr22a/b ${ }^{R F P}$ ) $\left(\mathrm{n}=6-10\right.$, females). Representative response traces to methyl hexanoate $\left(10^{-6}\right)$ and ethyl butanoate $\left(10^{-2}\right)$ are shown to the left.

f. Heat map representation of the data shown in e, together with the data of the DsimOr22awt response profile when expressed in $D$ secOr $22 a^{R F P}$ (replotted from Fig. 3c). The receptors expressed in the analysed neurons are listed to the right. Significant differences to $D$. simulans wild-type responses are shown (pairwise Wilcoxon rank-sum test and $P$ values adjusted for multiple comparisons using the $\mathrm{B} \& \mathrm{H}$ method): *** $P<0.001$; ** $P<0.01$; * $P$ $<0.05$; n.s. $P>0.05$. The equivalent responses to ethyl butanoate of $D$. simulans wild-type and Or22a mutant neurons (but complete loss in Or22a/b mutant neurons) suggests that this odour is detected principally by OR22b.

g, Boxplots with individual data points of the electrophysiological data presented in Fig. 3b. h, Boxplots with individual data points of the electrophysiological data presented in Fig. 3c. i, Boxplots with individual data points of the electrophysiological data presented in Fig. 3e. 

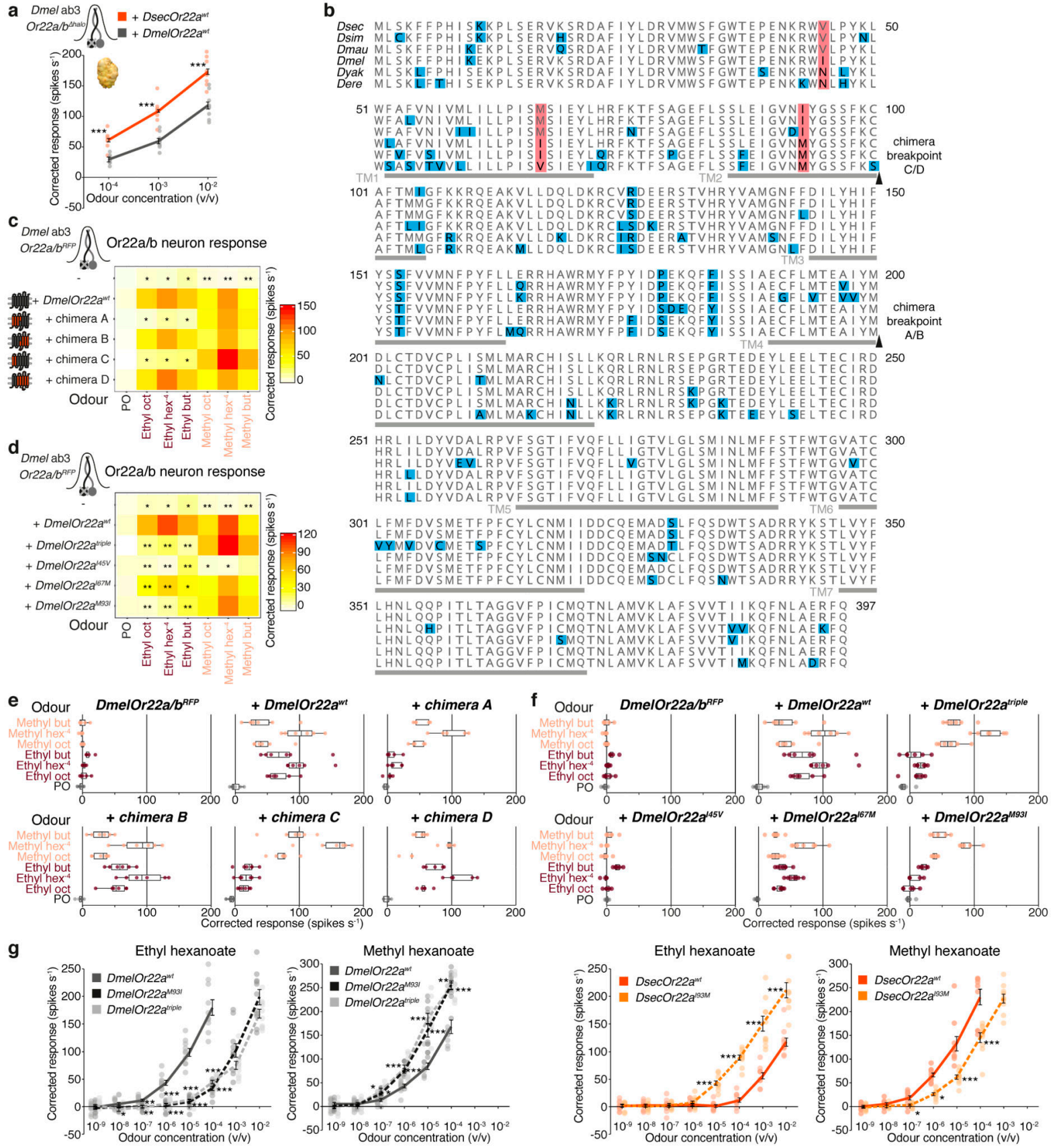

Extended Data Fig. 9. Mapping of odour-specificity determinants of OR22a.

a, Electrophysiological responses of $D$. melanogaster Or $22 a / b$ mutant neurons expressing $D s e c O r 22 a^{w t}$ or DmelOr22 $a^{w t}$ upon stimulation with increasing concentrations of noni fruit extract (mean \pm SEM and individual data points; $\mathrm{n}=9$, females). Significantly different values are indicated (pairwise Wilcoxon rank-sum test): $* * * P<0.001$.

b, Protein sequence alignment of OR22a orthologues of six species within the $D$. melanogaster species subgroup. Red shading: amino acid differences between $D$. melanogaster and $D$. sechellia, $D$. simulans and $D$. mauritiana that were analysed by 
mutagenesis in this study; blue shading: all other sequence differences. Arrowheads:

chimera breakpoints (see c). Predicted transmembrane (TM) domains are indicated with grey lines (location as in ${ }^{11}$ ).

c, Electrophysiological responses to a panel of noni odours conferred by chimeric OR22a proteins encoded by transgenes integrated at the Or22a/blocus of D. melanogaster $(\mathrm{n}=5-6$, females). Schematics on the left indicate the relative proportions of $D$. sechellia (red) and $D$. melanogaster (dark grey) sequences in each chimera (precise chimera breakpoints are shown in b). Significant differences to DmelOr $22 a^{w t}$ responses are shown (c, $\mathbf{d}$ and $\mathbf{g}$ : pairwise Wilcoxon rank-sum test and $P$ values adjusted for multiple comparisons using the B\&H method): *** $P<0.001$; ** $P<0.01$; $* P<0.05$; n.s. $P>0.05$.

d, Electrophysiological responses of $D$. melanogaster Or $22 a / b$ mutant neurons expressing different OR22a variants ( $\mathrm{n}=5-7$, females). The location of each mutated residue is indicated in b. Data for Or22a/b mutant and DmelOr $22 a^{w t}$ responses are replotted from c. Significant differences to DmelOr22a ${ }^{w t}$ responses are shown.

e, Boxplots with individual data points showing the same data as in $\mathbf{c}$.

f, Boxplots with individual data points showing the same data as in $\mathbf{d}$.

g, Dose-dependent electrophysiological responses of $D$. sechellia Or22a neurons expressing the indicated transgenes to ethyl or methyl hexanoate (mean \pm SEM and individual data points; $\mathrm{n}=10$, females). Significant comparisons to either the DmelOr22a ${ }^{w t}$ (left graphs) or the DsecOr22a ${ }^{w t}$ (right graphs) transgene responses are shown. 

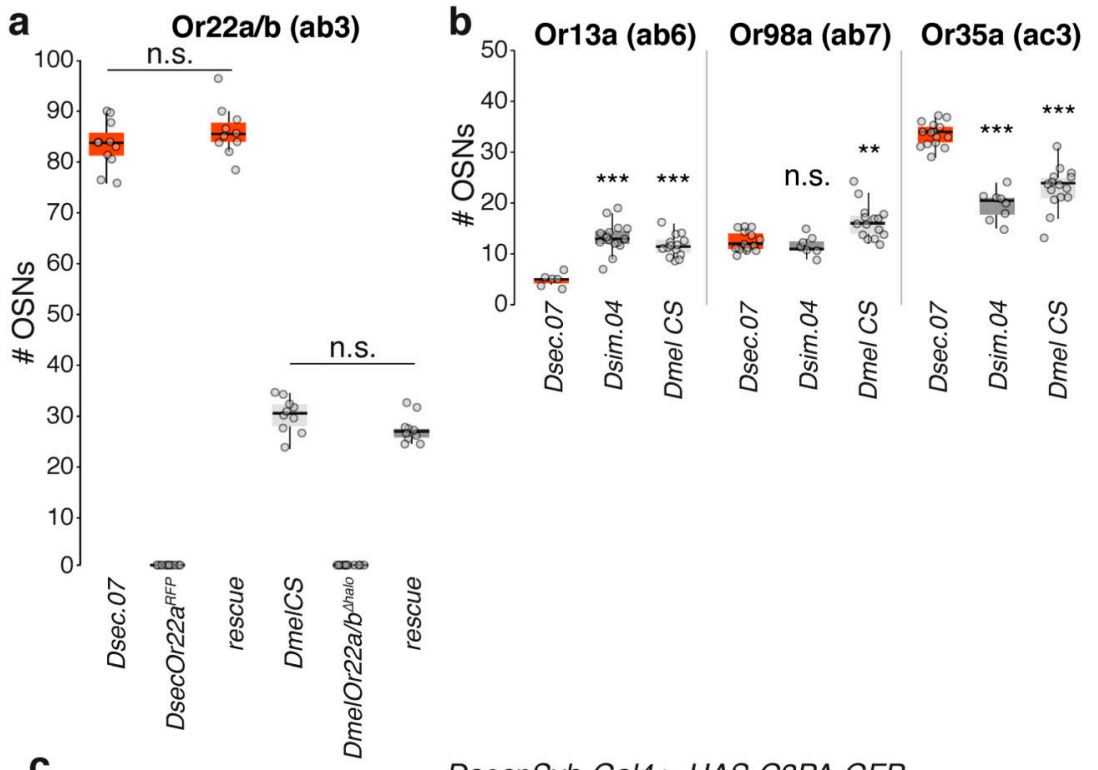

C
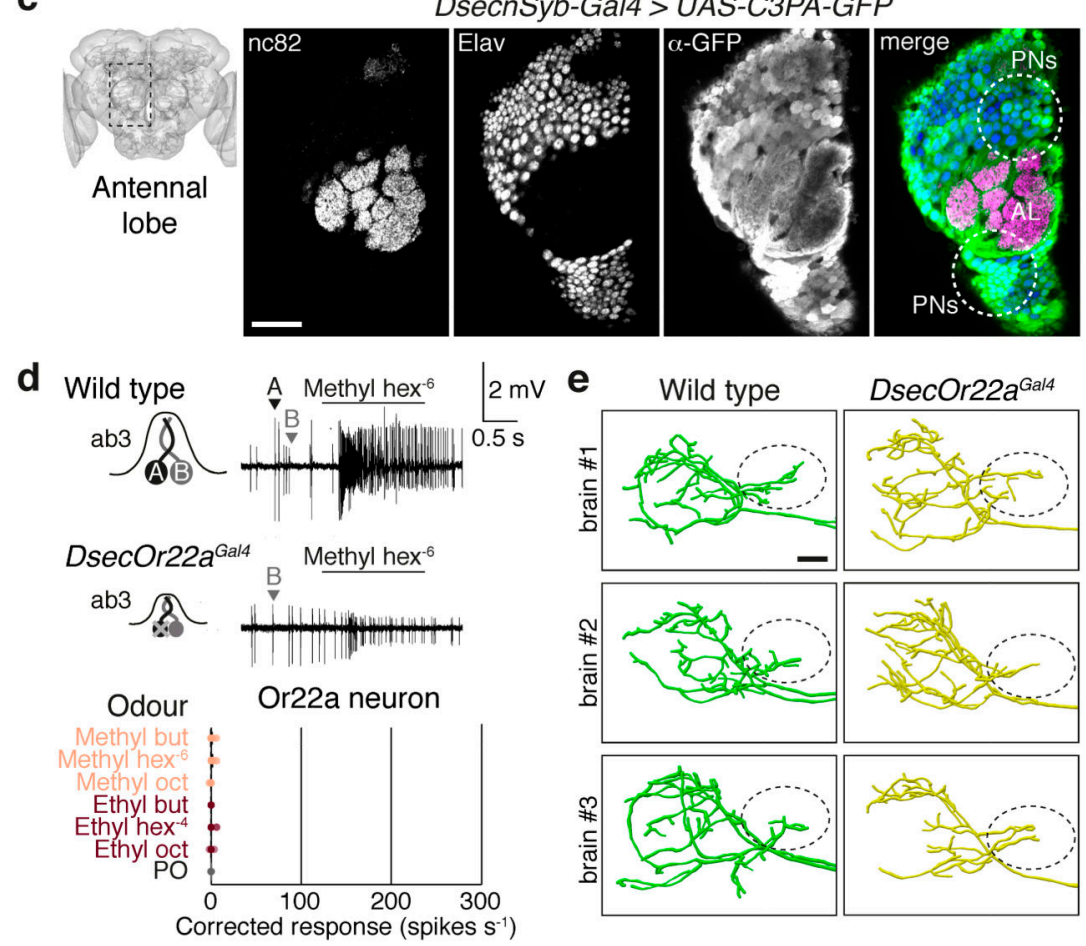

Extended Data Fig. 10. Peripheral and central olfactory circuit changes in D. sechellia.

a, Quantification of the number of OSNs expressing $\operatorname{Or} 22 \mathrm{a} /(\mathrm{b})$ in antennae of wild-type $D$. sechellia (DSSC 14021-0248.07) and D. melanogaster (Canton-S) (data as shown in Fig. 4b), Or22a/(b) mutants in both species, and rescue lines expressing $D \operatorname{secOr} 22 a^{w t}$ ( $\mathrm{n}=9-11$, females). Comparisons of rescue and wild-type genotypes for each species are shown (pairwise Wilcoxon rank-sum test). n.s. $P>0.05$. No significant differences in Or22a cell number were observed for different rescue transgenes (data not shown). 
b, Quantification of the number of OSNs expressing Or13a (ab6), Or98a (ab7) or Or35a (ac3I/II) in D. sechellia (DSSC 14021-0248.07), D. simulans (DSSC 14021-0251.004), and D. melanogaster (Canton-S) ( $\mathrm{n}=10-15$, females). Comparisons to Dsec.07 cell number counts are shown (pairwise Wilcoxon rank-sum test and $P$ values adjusted for multiple comparisons using the $\mathrm{B} \& \mathrm{H}$ method): *** $P<0.001$; ** $P<0.01$; n.s. $P>0.05$. c, Immunofluorescence with nc82 (neuropil), a-Elav (neurons) and a-GFP in a DsecnSybGal4/UAS-C3PA-GFP transgenic line, which expresses photoactivatable GFP panneuronally. The schematic on the left indicates the region of image acquisition. An anterior section through the antennal lobe (AL) is shown to reveal the position of the labelled projection neuron (PN) soma (circled in the right panel). Scale bar, $25 \mu \mathrm{m}$.

d, Electrophysiological responses of the Or22a neuron to odours present in noni in homozygous DsecOr22aGal4 (mutant) transgenic animals ( $\mathrm{n}=6$, females). Data points represent the solvent-corrected activities. Representative response traces to methyl hexanoate $\left(10^{-6}\right)$ in wild-type and transgenic animals are shown on top.

e, Tracing of axonal branches in the lateral horn of dye-filled DM2 PNs in D. sechellia wildtype and homozygous DsecOr22a Gal4 mutant flies. Three representative samples are shown. The circles depict the position of the $D$. sechellia-specific axonal branch. Scale bar, $10 \mu \mathrm{m}$. Samples could not be discriminated by genotype when presented to six independent researchers blindly. 


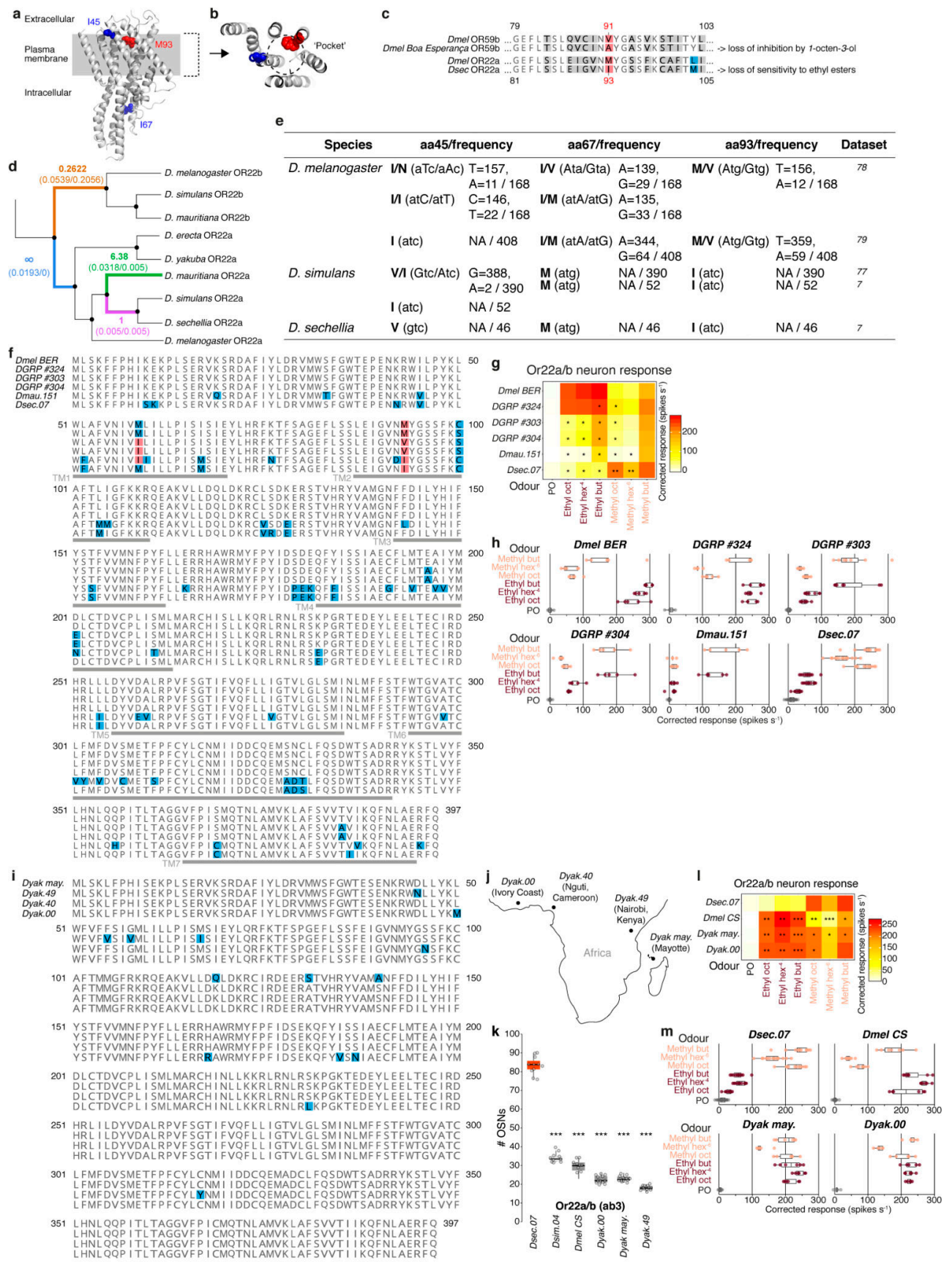

Extended Data Fig. 11. Phylogenetic and functional analysis of odour-specificity determinants in OR22a.

a, Side view of the ORCO monomer structure (determined by cryo-electronic microscopy ${ }^{37}$ ); the approximate location of the plasma membrane is indicated. The location of the residues corresponding to the odour-specificity determinants of OR22a analysed in this study (based on alignments generated $\mathrm{in}^{37}$ ) are highlighted as spheres.

b, Top view of a cross-section through the putative ligand-binding pocket of the ORCO structure shown in $\mathbf{a}$. 
c, Partial protein sequence alignment of OR22a and OR59b. The equivalent residue to $D$. melanogaster OR22a M93 in OR59b is V91, which exhibits intraspecific sequence variation impacting odour sensitivity ${ }^{82}$.

d, Results of branch-based models of molecular evolution that tested for changes in the rates of protein evolution among OR22a and OR22b orthologues (see Methods and

Supplementary Table 8): the rate of protein changes within the OR22a/OR22b phylogenetic tree highlights $\mathrm{dN} / \mathrm{dS}$ ratios $(\omega)$ that differ from the "background rate" $(\omega=0.1772)$. Most branches exhibited low $\omega$, arguing for strong purifying selection to maintain protein function over much of the tree. The two $\omega$ values that are $>1$ indicate an excess of protein changes, consistent with positive selection. The branch leading to $D$. simulans and $D$. sechellia OR22a displays nearly equal rates of silent and replacement substitutions, consistent with relaxed constraint during this period.

e, Allele frequencies within population datasets for D. melanogaster ${ }^{78,79}$, D. $_{\text {simulans }}{ }^{7,77}$ and $D$. sechellia ${ }^{7}$ at the three sites of OR22a that were functionally characterised in this study. The table displays amino acid (aa) positions 45, 67 and 93 of OR22a and the frequencies at which variants within the corresponding codons are segregating (number of alleles with respective variant/number of alleles analysed). "NA" (not applicable) indicates that positions within the codon are invariant. Datasets analysed are referenced on the right. Selected Or22a variants from the Drosophila melanogaster Genetic Reference Panel $(\text { DGRP })^{79}$ were confirmed by sequencing (f) and Or22a neuron physiology analysed (g,h). f, Protein sequence alignment of OR22a orthologues of $D$. melanogaster ${ }^{83}$, three lines of the DGRP, D. mauritiana (DSSC 14021-0241.151) and D. sechellia (DSSC 14021-0248.07).

Red shading: shared amino acid differences of DGRP \#303, DGRP \#304 and D. mauritiana (compared to other sequences) at position 59 and the key odour specificity-determinant at residue 93; blue shading: all other sequence differences. No line within the DGRP with a polymorphism only at position 93 was identified.

g, Electrophysiological responses of the Or22a/b neuron to odours present in noni $(\mathrm{n}=5-20$, females) in the strains shown in $\mathbf{f}$. The similarity between the response profiles of $D G R P$ \#303, DGRP\#304 and D. mauritiana suggests that their only shared polymorphism (at position 59) modifies OR22a response properties in these strains. Comparisons to Dmel $B E R$ responses are shown (pairwise Wilcoxon rank-sum test and $P$ values adjusted for multiple comparisons using the $\mathrm{B} \& \mathrm{H}$ method): $* * * P<0.001 ; * * P<0.01 ;$ n.s. $P>0.05$. $D$. mauritiana and $D$. sechellia data are replotted from Fig. $3 b$.

h, Boxplots with individual data points showing the same data as in g. D. mauritiana and $D$. sechellia data are replotted from Extended Data Fig. $8 \mathrm{~g}$.

i, Protein sequence alignment of OR22a orthologues of the noni-specialised D. yakuba mayottensis $^{41}$ (Dyak may.) and three other D. yakuba strains (DSSC 14021-0261.00, 14021-0261.40, 14021-0261.49). Blue shading: differences between these sequences. $\mathbf{j}$, Collection sites of D. yakuba strains shown in $\mathbf{i}$.

$\mathbf{k}$, Quantification of the number of OSNs expressing Or22a/(b) in D. sechellia (DSSC 14021-0248.07), D. simulans (DSSC 14021-0251.004), D. melanogaster (Canton-S) (data as shown in Fig. 4b), D. yakuba (DSSC 14021-0261.00, 14021-0261.49) and D. yakuba mayottensis ( $\mathrm{n}=10-12$, females). Comparisons to Dsec.07 cell number counts are shown (k and $\mathbf{I}$ : pairwise Wilcoxon rank-sum test and $P$ values adjusted for multiple comparisons using the B\&H method): *** $P<0.001$; ** $P<0.01$; $* P<0.05$; n.s. $P>0.05$. 
1, Electrophysiological responses to odours present in noni of the Or22a/(b) neurons in $D$. sechellia (DSSC 14021-0248.07), D. melanogaster (Canton-S), D. yakuba mayottensis ( $\mathrm{n}=$ 5-20, females) and D. yakuba (DSSC 14021-0261.00). Comparisons to Dsec.O7 responses are shown. D. sechellia, D. melanogaster and D. yakuba.00 data are replotted from Fig. 3b. $\mathbf{m}$, Boxplots with individual data points showing the same data as in $\mathbf{l}$. D. sechellia, $D$. melanogaster and D. yakuba.00 data are replotted from Extended Data Fig. 8g. 


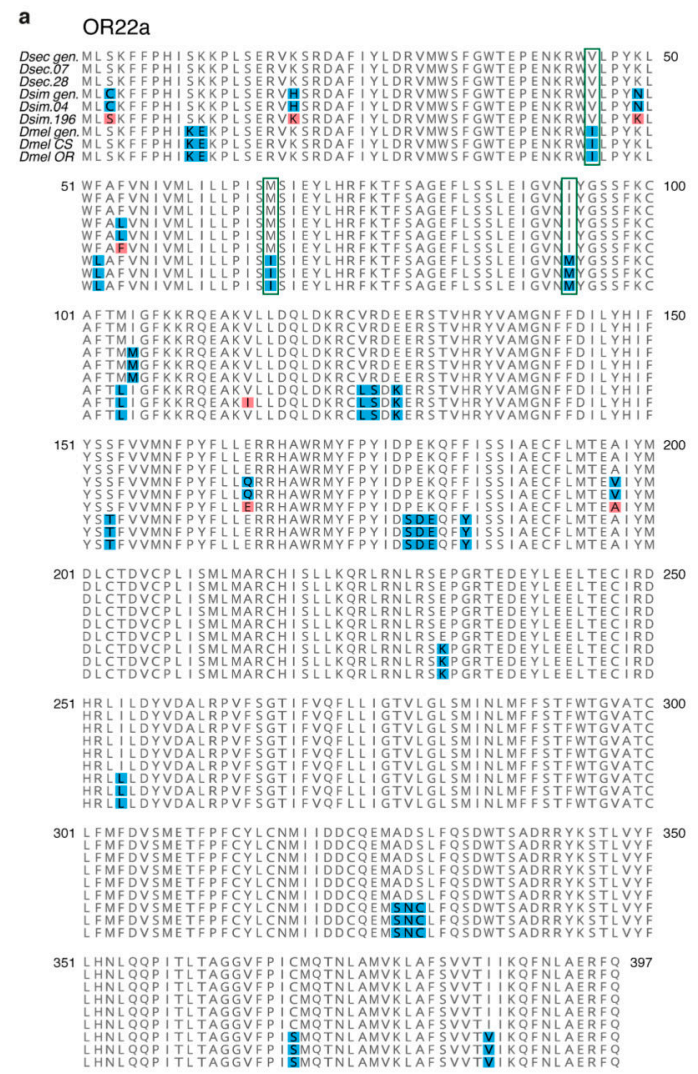

b IR75b

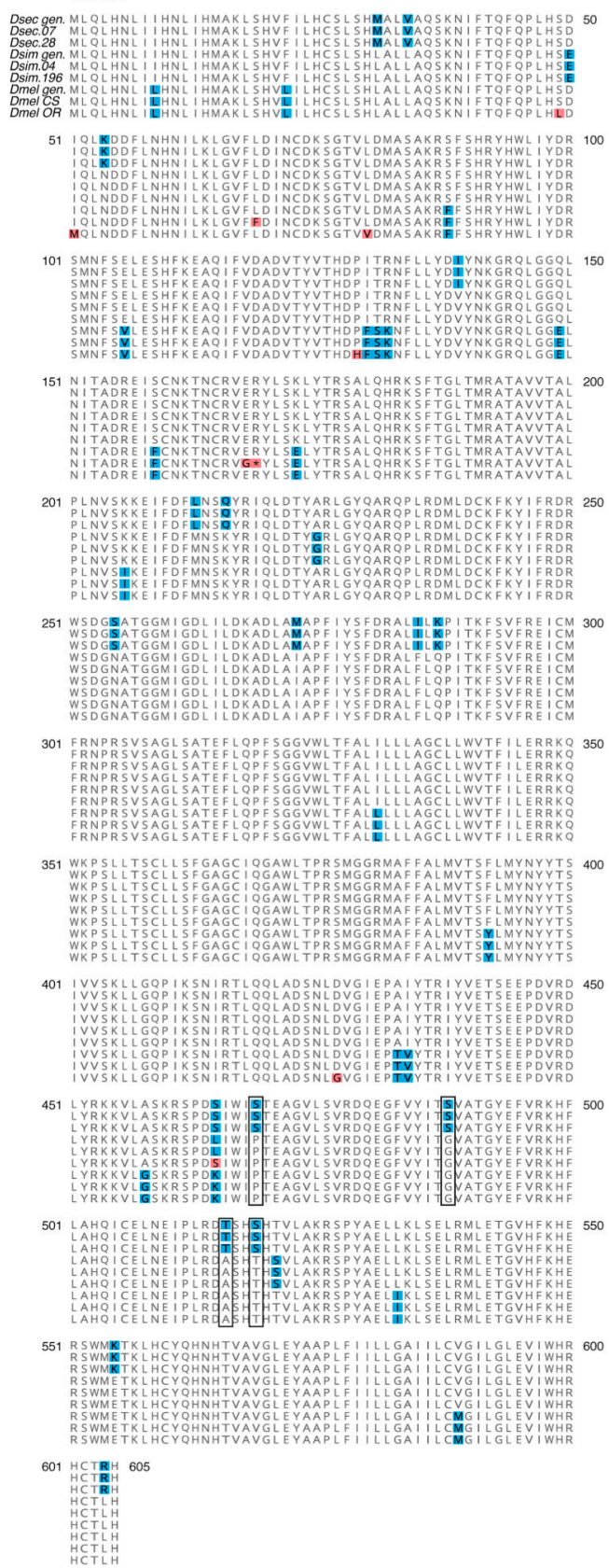

Extended Data Fig. 12. Protein sequence alignments of OR22a and IR75b.

a, OR22a orthologues of strains used for behavioural assays as well as genome-sequenced strains ("gen.") of each species (version: D. sechellia (r1.3), D. simulans (r2.01), D. melanogaster(r6.28)). Blue/red shading: differences between species/strains. Green boxes: residues tested in this study for their role in defining ester tuning specificity.

b, IR75b orthologues of same strains as in a. Blue/red shading: differences between species/ strains. Black boxes: residues predicted to be located within the ligand-binding domain that contribute to odour tuning specificity ${ }^{63}$. Note that the premature stop codon of the Canton-S 
strain (position 169, marked by an asterisk) does not impair receptor function, as shown in other strains ${ }^{63}$.

\section{Supplementary Material}

Refer to Web version on PubMed Central for supplementary material.

\section{Acknowledgements}

We thank Y. Bellaïche, B. Deplancke, K. S. Douglas, S. Lavista-Llanos, K. O’Connor-Giles, J. Posakony, V. Ruta, D. Stern, G. Suh, A. Yassin, the Bloomington Drosophila Stock Center (NIH P40OD018537) and the Developmental Studies Hybridoma Bank (NICHD of the NIH, University of Iowa) for reagents, B. Prud'homme for instruction on Drosophila microinjections, B. Sutcliffe and S. Cachero for advice on the generation of reference brains, I. Alali and K. Weniger for technical assistance, J. Simpson for details of the $n S y b$ promoter construct and P. C. Chai for assistance with glomerular identification. We thank I. Rentero Rebollo, J. Sánchez-Alcañiz, L. PrietoGodino and members of the Benton laboratory for discussions and comments on the manuscript. T.O.A. is supported by a Human Frontier Science Program Long-Term Fellowship (LT000461/2015-L). M.A.K., B.S.H., and M.K. are supported by the Max Planck Society. K.E. and S.J.C.C. are supported by a National Institute of Health Award (1 R01 NS 167970) and a Eunice Kennedy Shriver National Institute of Child Health \& Human Development Award of the National Institutes of Health (5 T32 HD 007491). J.R.A. is supported by a Swiss National Science Professorship Grant (PP00P3 176956). G.S.X.E.J. is supported by an ERC Consolidator Grant (649111) and the MRC (MC_U105188491). Research in R.B.'s laboratory is supported by ERC Consolidator and Advanced Grants (615094 and 833548, respectively), the Swiss National Science Foundation and the Fondation Herbette.

\section{References}

1. Arguello JR, Benton R. Open questions: Tackling Darwin's "instincts": the genetic basis of behavioural evolution. BMC Biol. 2017; 15:26. [PubMed: 28372547]

2. Bendesky A, Bargmann CI. Genetic contributions to behavioural diversity at the gene-environment interface. Nat Rev Genet. 2011; 12:809-820. [PubMed: 22064512]

3. Bendesky A, et al. The genetic basis of parental care evolution in monogamous mice. Nature. 2017; 544:434-439. [PubMed: 28424518]

4. Ding Y, Berrocal A, Morita T, Longden KD, Stern DL. Natural courtship song variation caused by an intronic retroelement in an ion channel gene. Nature. 2016; 536:329-332. [PubMed: 27509856]

5. Weber JN, Peterson BK, Hoekstra HE. Discrete genetic modules are responsible for complex burrow evolution in Peromyscus mice. Nature. 2013; 493:402-405. [PubMed: 23325221]

6. Garrigan D, et al. Genome sequencing reveals complex speciation in the Drosophila simulans clade. Genome Res. 2012; 22:1499-1511. [PubMed: 22534282]

7. Schrider DR, Ayroles J, Matute DR, Kern AD. Supervised machine learning reveals introgressed loci in the genomes of Drosophila simulans and D. sechellia. Plos Genetics. 2018; 14

8. Amlou M, Moreteau B, David JR. Genetic analysis of Drosophila sechellia specialization: Oviposition behaviour toward the major aliphatic acids of its host plant. Behav Genet. 1998; 28:455-464. [PubMed: 9926614]

9. Cobb M, Burnet B, Blizard R, Jallon JM. Courtship in Drosophila sechellia - its structure, functional aspects, and relationship to those of other members of the Drosophila melanogaster species subgroup. J Insect Behav. 1989; 2:63-89.

10. Coyne JA. Genetics of sexual isolation in females of the Drosophila simulans species complex. Genet Res. 1992; 60:25-31. [PubMed: 1452013]

11. Dekker T, Ibba I, Siju KP, Stensmyr MC, Hansson BS. Olfactory shifts parallel superspecialism for toxic fruit in Drosophila melanogaster sibling, D. sechellia. Curr Biol. 2006; 16:101-109. [PubMed: 16401429]

12. Higa I, Fuyama Y. Genetics of Food Preference in Drosophila sechellia. 1. Responses to Food Attractants. Genetica. 1993; 88:129-136. [PubMed: 8224853] 
13. Ibba I, Angioy AM, Hansson BS, Dekker T. Macroglomeruli for fruit odours change blend preference in Drosophila. Die Naturwissenschaften. 2010; 97:1059-1066. [PubMed: 20972770]

14. Matsuo T, Sugaya S, Yasukawa J, Aigaki T, Fuyama Y. Odorant-binding proteins OBP57d and OBP57e affect taste perception and host-plant preference in Drosophila sechellia. PLoS Biol. 2007; 5:e118. [PubMed: 17456006]

15. Prieto-Godino LL, et al. Evolution of acid-sensing olfactory circuits in Drosophilids. Neuron. 2017; 93:661-676. [PubMed: 28111079]

16. R'Kha S, Capy P, David JR. Host-plant specialization in the Drosophila melanogaster species complex: a physiological, behavioural, and genetical analysis. Proc Natl Acad Sci U S A. 1991; 88:1835-1839. [PubMed: 1900368]

17. Yalcin B, et al. Genetic dissection of a behavioural quantitative trait locus shows that Rgs2 modulates anxiety in mice. Nat Genet. 2004; 36:1197-1202. [PubMed: 15489855]

18. Bendesky A, Tsunozaki M, Rockman MV, Kruglyak L, Bargmann CI. Catecholamine receptor polymorphisms affect decision-making in C. elegans. Nature. 2011; 472:313-318. [PubMed: 21412235]

19. Bumbarger DJ, Riebesell M, Rodelsperger C, Sommer RJ. System-wide rewiring underlies behavioral differences in predatory and bacterial-feeding nematodes. Cell. 2013; 152:109-119. [PubMed: 23332749]

20. Markow TA, O'Grady P. Reproductive ecology of Drosophila. Functional Ecology. 2008; 22:747759.

21. Linz J, et al. Host plant-driven sensory specialization in Drosophila erecta. Proc Biol Sci. 2013; 280

22. Seeholzer LF, Seppo M, Stern DL, Ruta V. Evolution of a central neural circuit underlies Drosophila mate preferences. Nature. 2018; 559:564-569. [PubMed: 29995860]

23. Dweck HK, et al. Olfactory channels associated with the Drosophila maxillary palp mediate shortand long-range attraction. Elife. 2016; 5

24. Matute DR, Ayroles JF. Hybridization occurs between Drosophila simulans and D. sechellia in the Seychelles archipelago. J Evol Biol. 2014; 27:1057-1068. [PubMed: 24773151]

25. Vosshall LB, Stocker RF. Molecular architecture of smell and taste in Drosophila. Annu Rev Neurosci. 2007; 30:505-533. [PubMed: 17506643]

26. Larsson MC, et al. Or83b encodes a broadly expressed odorant receptor essential for Drosophila olfaction. Neuron. 2004; 43:703-714. [PubMed: 15339651]

27. Abuin L, et al. Functional architecture of olfactory ionotropic glutamate receptors. Neuron. 2011; 69:44-60. [PubMed: 21220098]

28. Benton R, Sachse S, Michnick SW, Vosshall LB. Atypical membrane topology and heteromeric function of Drosophila odorant receptors in vivo. PLoS Biol. 2006; 4:e20. [PubMed: 16402857]

29. Stensmyr MC, Dekker T, Hansson BS. Evolution of the olfactory code in the Drosophila melanogaster subgroup. Proc Biol Sci. 2003; 270:2333-2340. [PubMed: 14667348]

30. Dobritsa AA, van der Goes van Naters W, Warr CG, Steinbrecht RA, Carlson JR. Integrating the molecular and cellular basis of odour coding in the Drosophila antenna. Neuron. 2003; 37:827841. [PubMed: 12628173]

31. Couto A, Alenius M, Dickson BJ. Molecular, anatomical, and functional organization of the Drosophila olfactory system. Curr Biol. 2005; 15:1535-1547. [PubMed: 16139208]

32. Shiao MS, et al. Expression divergence of chemosensory genes between Drosophila sechellia and its sibling species and its implications for host shift. Genome Biol Evol. 2015; 7:2843-2858. [PubMed: 26430061]

33. Yao CA, Ignell R, Carlson JR. Chemosensory coding by neurons in the coeloconic sensilla of the Drosophila antenna. J Neurosci. 2005; 25:8359-8367. [PubMed: 16162917]

34. Ai M, et al. Acid sensing by the Drosophila olfactory system. Nature. 2010; 468:691-695. [PubMed: 21085119]

35. Ruta V, et al. A dimorphic pheromone circuit in Drosophila from sensory input to descending output. Nature. 2010; 468:686-690. [PubMed: 21124455]

36. Grabe V, Sachse S. Fundamental principles of the olfactory code. Bio Systems. 2018; 164:94-101. 
37. Butterwick JA, et al. Cryo-EM structure of the insect olfactory receptor ORCO. Nature. 2018; 560:447-452. [PubMed: 30111839]

38. Aguade M. Nucleotide and copy-number polymorphism at the odorant receptor genes Or22a and Or22b in Drosophila melanogaster. Mol Biol Evol. 2009; 26:61-70. [PubMed: 18922763]

39. Goldman-Huertas B, et al. Evolution of herbivory in Drosophilidae linked to loss of behaviours, antennal responses, odorant receptors, and ancestral diet. Proc Natl Acad Sci U S A. 2015; 112:3026-3031. [PubMed: 25624509]

40. Nozawa M, Nei M. Evolutionary dynamics of olfactory receptor genes in Drosophila species. Proc Natl Acad Sci U S A. 2007; 104:7122-7127. [PubMed: 17438280]

41. Yassin A, et al. Recurrent specialization on a toxic fruit in an island Drosophila population. Proc Natl Acad Sci U S A. 2016; 113:4771-4776. [PubMed: 27044093]

42. Ambrose D, Ellender JH, Lee EB, Sprake CHS, Townsend R. Thermodynamic properties of organic oxygen compounds XXXVIII. Vapour pressures of some aliphatic ketones. The Journal of Chemical Thermodynamics. 1975; 7:453-472.

43. Daubert TE, Danner RP. Physical and thermodynamic properties of pure chemicals data compilation. Data compilation. 1997

44. Port F, Chen HM, Lee T, Bullock SL. Optimized CRISPR/Cas tools for efficient germline and somatic genome engineering in Drosophila. Proc Natl Acad Sci U S A. 2014

45. Port F, Bullock SL. Augmenting CRISPR applications in Drosophila with tRNA-flanked sgRNAs. Nat Methods. 2016; 13:852-854. [PubMed: 27595403]

46. Barolo S, Carver LA, Posakony JW. GFP and beta-galactosidase transformation vectors for promoter/enhancer analysis in Drosophila. Biotechniques. 2000; 29:726-732. [PubMed: 11056799]

47. Gratz SJ, et al. Highly specific and efficient CRISPR/Cas9-catalyzed homology-directed repair in Drosophila. Genetics. 2014; 196:961-971. [PubMed: 24478335]

48. Stern DL. Tagmentation-Based Mapping (TagMap) of Mobile DNA Genomic Insertion Sites. bioRxiv. 2016; doi: 10.1101/037762

49. Croset V, et al. Ancient protostome origin of chemosensory ionotropic glutamate receptors and the evolution of insect taste and olfaction. PLoS Genet. 2010; 6:e1001064. [PubMed: 20808886]

50. Bateman JR, Wu CT. A simple polymerase chain reaction-based method for the construction of recombinase-mediated cassette exchange donor vectors. Genetics. 2008; 180:1763-1766. [PubMed: 18791223]

51. Groth AC, Olivares EC, Thyagarajan B, Calos MP. A phage integrase directs efficient site-specific integration in human cells. Proc Natl Acad Sci U S A. 2000; 97:5995-6000. [PubMed: 10801973]

52. Bischof J, Maeda RK, Hediger M, Karch F, Basler K. An optimized transgenesis system for Drosophila using germ-line-specific phiC31 integrases. Proc Natl Acad Sci U S A. 2007; 104:3312-3317. [PubMed: 17360644]

53. Han C, Jan LY, Jan YN. Enhancer-driven membrane markers for analysis of nonautonomous mechanisms reveal neuron-glia interactions in Drosophila. Proc Natl Acad Sci U S A. 2011; 108:9673-9678. [PubMed: 21606367]

54. Arnoult L, et al. Emergence and diversification of fly pigmentation through evolution of a gene regulatory module. Science. 2013; 339:1423-1426. [PubMed: 23520110]

55. Gratz SJ, et al. Genome engineering of Drosophila with the CRISPR RNA-guided Cas 9 nuclease. Genetics. 2013; 194:1029-1035. [PubMed: 23709638]

56. Zhang X, Koolhaas WH, Schnorrer F. A versatile two-step CRISPR- and RMCE-based strategy for efficient genome engineering in Drosophila. G3 (Bethesda). 2014; 4:2409-2418. [PubMed: 25324299]

57. Gohl DM, et al. A versatile in vivo system for directed dissection of gene expression patterns. Nat Methods. 2011; 8:231-237. [PubMed: 21473015]

58. Knecht ZA, et al. Distinct combinations of variant ionotropic glutamate receptors mediate thermosensation and hygrosensation in Drosophila. Elife. 2016; 5

59. Silbering AF, et al. Complementary function and integrated wiring of the evolutionarily distinct Drosophila olfactory subsystems. J Neurosci. 2011; 31:13357-13375. [PubMed: 21940430] 
60. Schindelin J, et al. Fiji: an open-source platform for biological-image analysis. Nat Methods. 2012; 9:676-682. [PubMed: 22743772]

61. Benton R, Dahanukar A. Electrophysiological recording from Drosophila olfactory sensilla. Cold Spring Harb Protoc. 2011; 2011:824-838. [PubMed: 21724819]

62. Saina M, Benton R. Visualizing olfactory receptor expression and localization in Drosophila. Methods Mol Biol. 2013; 1003:211-228. [PubMed: 23585045]

63. Prieto-Godino LL, et al. Olfactory receptor pseudo-pseudogenes. Nature. 2016; 539:93-97. [PubMed: 27776356]

64. Benton R, Vannice KS, Gomez-Diaz C, Vosshall LB. Variant ionotropic glutamate receptors as chemosensory receptors in Drosophila. Cell. 2009; 136:149-162. [PubMed: 19135896]

65. Sanchez-Alcaniz JA, Zappia G, Marion-Poll F, Benton R. A mechanosensory receptor required for food texture detection in Drosophila. Nat Commun. 2017; 8

66. Ostrovsky A, Cachero S, Jefferis G. Clonal analysis of olfaction in Drosophila: immunochemistry and imaging of fly brains. Cold Spring Harb Protoc. 2013; 2013:342-346. [PubMed: 23547149]

67. Jefferis GS, et al. Comprehensive maps of Drosophila higher olfactory centers: spatially segregated fruit and pheromone representation. Cell. 2007; 128:1187-1203. [PubMed: 17382886]

68. Manton JD, et al. Combining genome-scale Drosophila 3D neuroanatomical data by bridging template brains. bioRxiv. 2014; doi: 10.1101/006353

69. Cachero S, Ostrovsky AD, Yu JY, Dickson BJ, Jefferis GS. Sexual dimorphism in the fly brain. Curr Biol. 2010; 20:1589-1601. [PubMed: 20832311]

70. Feng L, Zhao T, Kim J. neuTube 1.0: A New Design for Efficient Neuron Reconstruction Software Based on the SWC Format. eNeuro. 2015; 2

71. Caron SJ, Ruta V, Abbott LF, Axel R. Random convergence of olfactory inputs in the Drosophila mushroom body. Nature. 2013; 497:113-117. [PubMed: 23615618]

72. Camacho C, et al. BLAST+: architecture and applications. BMC Bioinformatics. 2009; 10:421. [PubMed: 20003500]

73. Birney E, Clamp M, Durbin R. GeneWise and Genomewise. Genome Res. 2004; 14:988-995. [PubMed: 15123596]

74. Abascal F, Zardoya R, Telford MJ. TranslatorX: multiple alignment of nucleotide sequences guided by amino acid translations. Nucleic Acids Res. 2010; 38:W7-13. [PubMed: 20435676]

75. Ronquist F, et al. MrBayes 3.2: efficient Bayesian phylogenetic inference and model choice across a large model space. Syst Biol. 2012; 61:539-542. [PubMed: 22357727]

76. Yang Z. PAML 4: phylogenetic analysis by maximum likelihood. Mol Biol Evol. 2007; 24:15861591. [PubMed: 17483113]

77. Signor SA, New FN, Nuzhdin S. A large panel of Drosophila simulans reveals an abundance of common variants. Genome Biol Evol. 2018; 10:189-206. [PubMed: 29228179]

78. Grenier JK, et al. Global diversity lines - a five-continent reference panel of sequenced Drosophila melanogaster strains. G3 (Bethesda). 2015; 5:593-603. [PubMed: 25673134]

79. Huang W, et al. Natural variation in genome architecture among 205 Drosophila melanogaster Genetic Reference Panel lines. Genome Res. 2014; 24:1193-1208. [PubMed: 24714809]

80. Danecek P, et al. The variant call format and VCFtools. Bioinformatics. 2011; 27:2156-2158. [PubMed: 21653522]

81. Grabe V, et al. Elucidating the neuronal architecture of olfactory glomeruli in the Drosophila antennal lobe. Cell Rep. 2016; 16:3401-3413. [PubMed: 27653699]

82. Pellegrino M, Steinbach N, Stensmyr MC, Hansson BS, Vosshall LB. A natural polymorphism alters odour and DEET sensitivity in an insect odorant receptor. Nature. 2011; 478:511-514. [PubMed: 21937991]

83. Adams MD, et al. The genome sequence of Drosophila melanogaster. Science. 2000; 287:21852195. [PubMed: 10731132] 

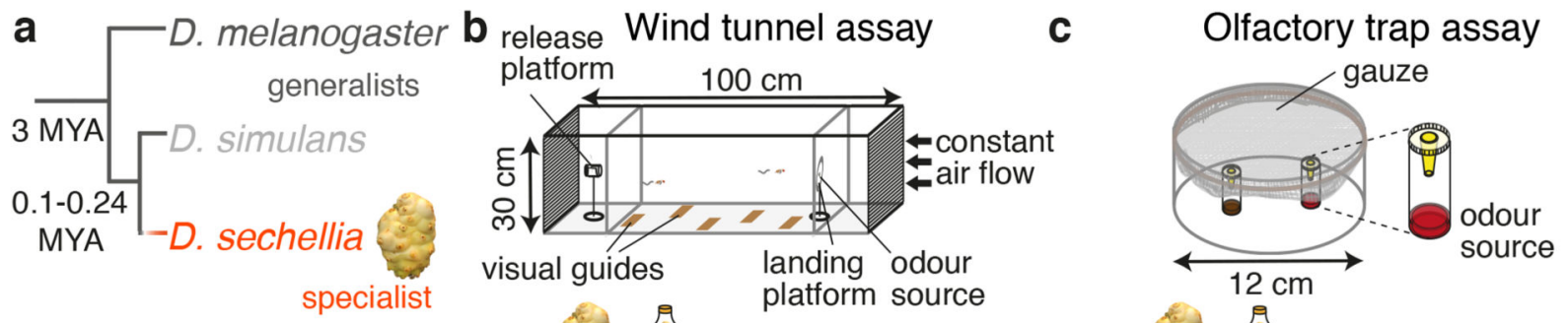

\section{d Noni volatiles}

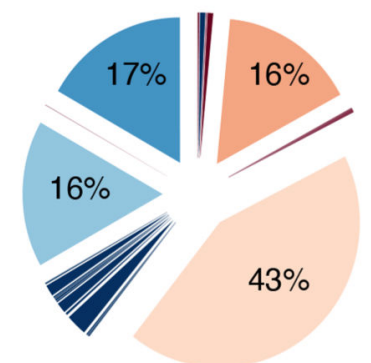

Other esters Methyl hexanoate Methyl octanoate Hexanoic acid Octanoic acid Others
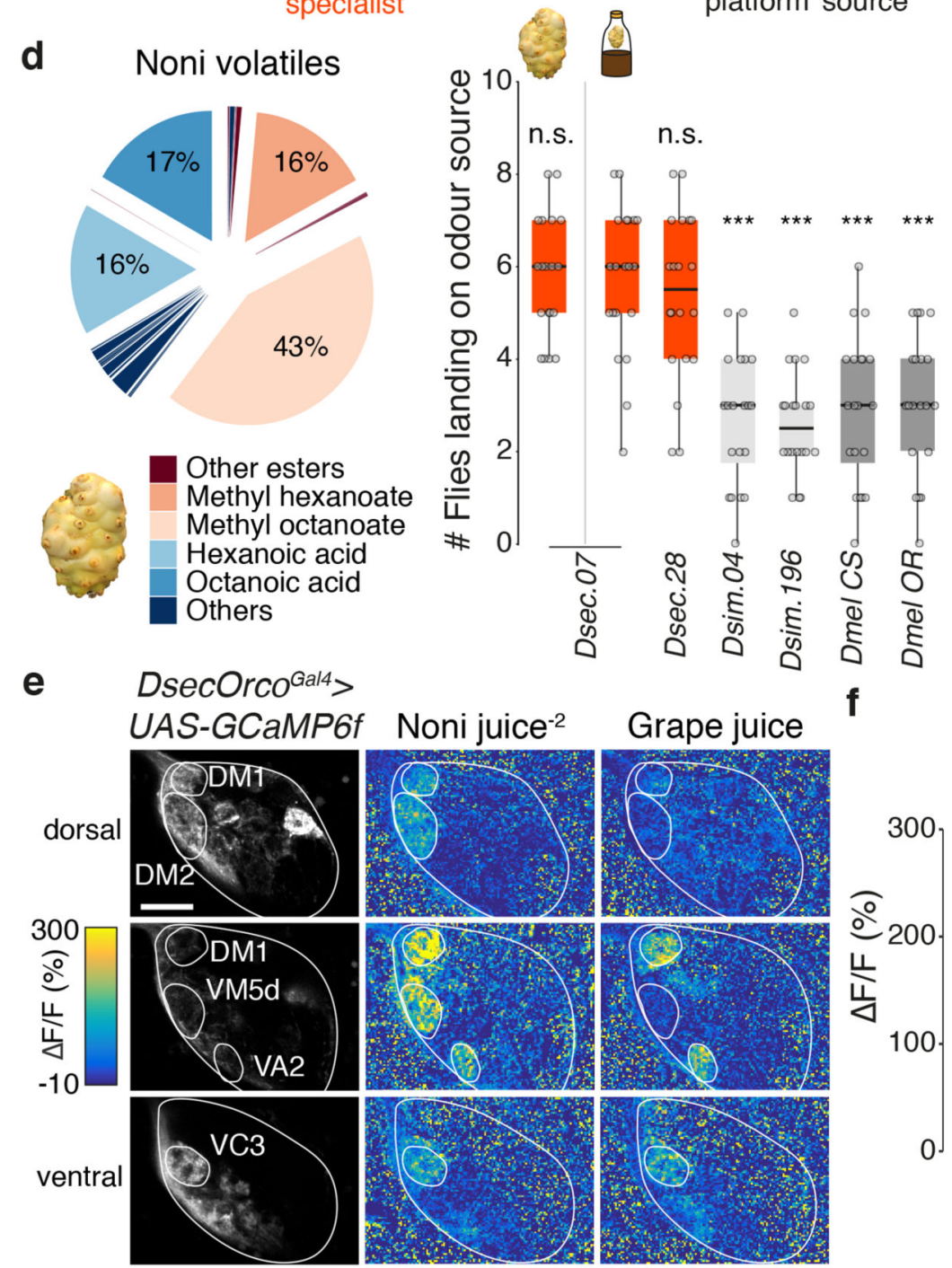

\section{DsecOrco ${ }^{\text {Gal4 }}>$ UAS-GCaMP6f}

\# Noni juice ${ }^{-2}$ 由 Grape juice n.s.

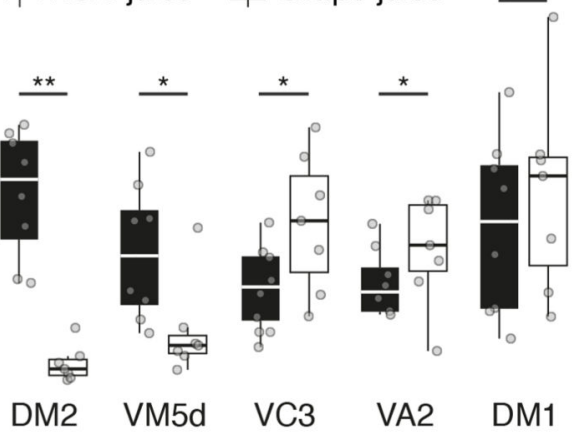

Glomerulus

Fig. 1. Behavioural and physiological responses of $D$. sechellia to noni.

a, $D$. sechellia specialises on noni fruit (Morinda citrifolia) while $D$. simulans and $D$. melanogaster are food generalists (MYA = million years ago).

b, Behavioural responses to noni fruit or juice in a wind tunnel assay of $D$. sechellia, $D$. simulans and D. melanogaster wild-type strains ( $\mathrm{n}=20$ experiments (10 females/ experiment)). In this and other panels, boxplots show the median, first and third quartile of the data, overlaid with individual data points. Comparisons to Dsec.07 noni juice responses are shown (Kruskal-Wallis test, Dunn's post-hoc correction): *** $P<0.001$; n.s. $P>0.05$. 
c, Behavioural responses in a trap assay testing preferences between noni and grape, or between noni juice and grape juice (same strains as in $\mathbf{b}, \mathrm{n}=15-27$ experiments, exact $\mathrm{n}$ in Source Data, (22-25 females/experiment)). Comparisons to Dsec.07 noni juice responses are shown (pairwise Wilcoxon rank-sum test, $P$ values adjusted for multiple comparisons using the Benjamini and Hochberg (B\&H) method): *** $P<0.001$; n.s. $P>0.05$.

d, Odour bouquet of a ripe noni fruit determined by gas chromatography/mass spectrometry (see Methods, Extended Data Fig. 2, Supplementary Table 1).

e, Representative odour-evoked calcium responses in the axon termini of Orco OSNs in the D. sechellia antennal lobe (genotype: UAS-GCaMP6f/UAS-GCaMP6f;;DsecOrcoGal4/+) acquired by two-photon imaging. Three focal planes are shown, revealing different glomeruli (outlined) along the dorsoventral axis. Left column: raw fluorescence images. Right columns: relative increase in GCaMP6f fluorescence $(\Delta \mathrm{F} / \mathrm{F} \%)$ after stimulation with noni juice $\left(10^{-2}\right.$ in $\left.\mathrm{H}_{2} \mathrm{O}\right)$ or grape juice. Scale bar, $25 \mu \mathrm{m}$.

f, Quantification of responses for the animals represented in e. Maximum response amplitudes for each experiment are plotted ( $\mathrm{n}=7-10$ females). Wilcoxon signed-rank test: ** $P<0.01 ; * P<0.05$; n.s. $P>0.05$. 


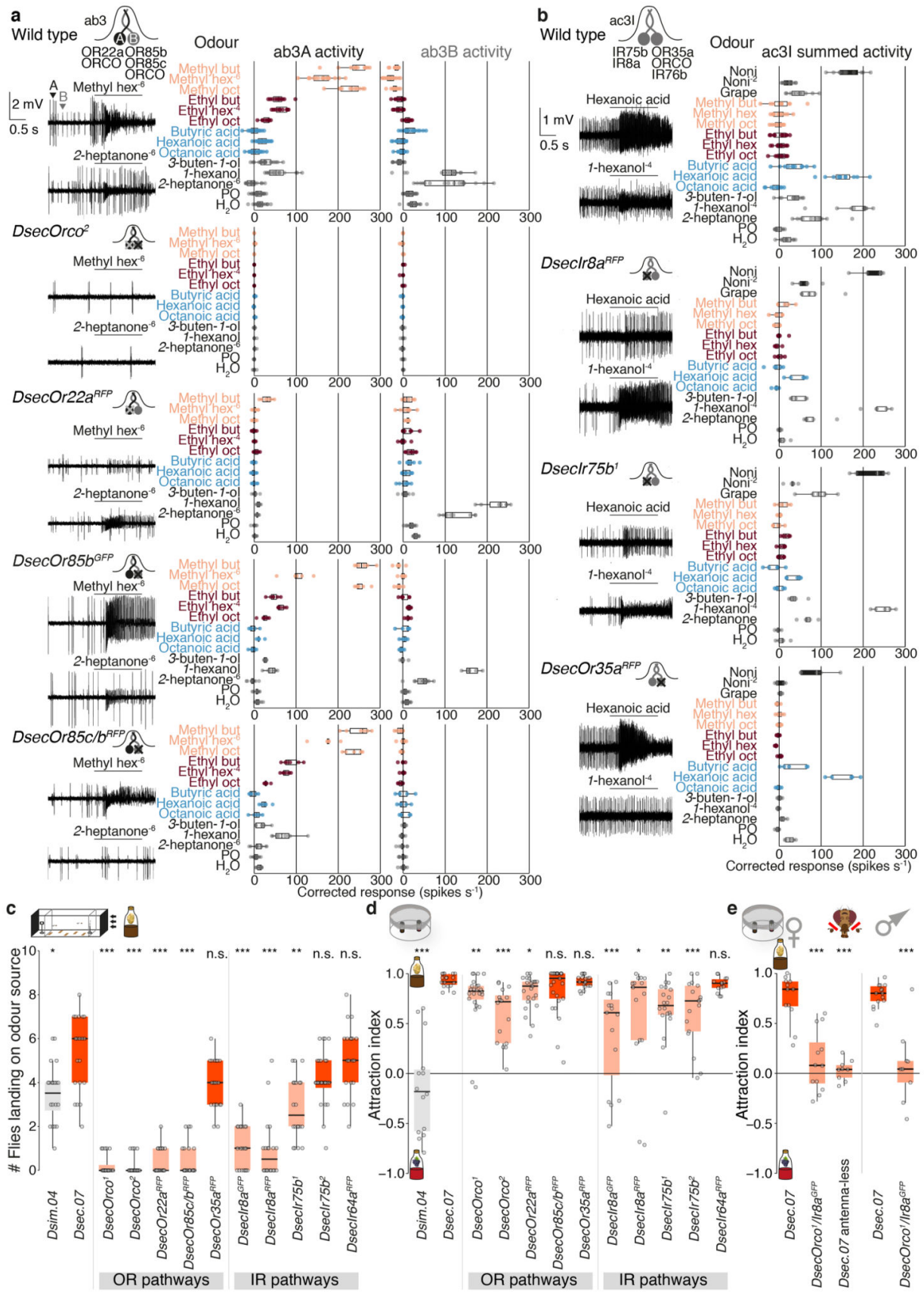

Fig. 2. Olfactory receptors contribution to noni-sensing.

a, Electrophysiological responses of ab3 sensillum neurons to noni odours ( $n=5-20$, females, see Supplementary Table 7 for exact $\mathrm{n}$ and mean spike counts) in wild-type and receptor mutant $D$. sechellia (schematised in the cartoons), with representative traces for methyl hexanoate and 2-heptanone. Data points are the solvent-corrected activities of individual neurons (arrowheads in wild-type traces). Odours (oct, octanoate; hex, hexanoate; but, butanoate) are coloured according to chemical class: methyl esters (salmon), ethyl esters 
(dark red), acids (light blue), others (black), used at $10^{-2} \mathrm{v} / \mathrm{v}$ unless indicated otherwise. PO $=$ paraffin oil.

b, Responses of ac3I neurons to noni juice, grape juice and noni odours ( $\mathrm{n}=5-11$, females) in wild-type and receptor mutant $D$. sechellia, with representative traces for hexanoic acid and 1-hexanol. Data points are the summed, solvent-corrected activities of both neurons. c, Behavioural responses to noni juice in the wind tunnel assay ( $\mathrm{n}=20$ experiments). Comparisons to Dsec.07 responses are shown (Kruskal-Wallis test, Dunn's post-hoc correction). c, d, e: red = no significant difference; salmon = significantly different response; *** $P<0.001$; ** $P<0.01$; * $P<0.05$; n.s. $P>0.05$.

d, Behavioural responses in the trap assay testing preference for noni juice or grape juice (n =13-25 experiments). Comparisons to Dsec.07 responses are shown ( $\mathbf{d}$ and $\mathbf{e}$, pairwise Wilcoxon rank-sum test, $P$ values adjusted for multiple comparisons using the B\&H method).

e, Behavioural responses in the trap assay testing preference for noni juice or grape juice using wild-type $D$. sechellia, DsecOrco ${ }^{1} / I r 8 a^{G F P}$ double mutants and antenna-less $D$. sechellia $(\mathrm{n}=9-15$ experiments (22-25 females or males, as indicated/experiment)). Average attraction indices for $\mathrm{DsecOrco}^{1} / \mathrm{Ir} 8 \mathrm{a}^{G F P}$ and antenna-less flies are not significantly different from zero (pairwise Wilcoxon rank-sum test). 


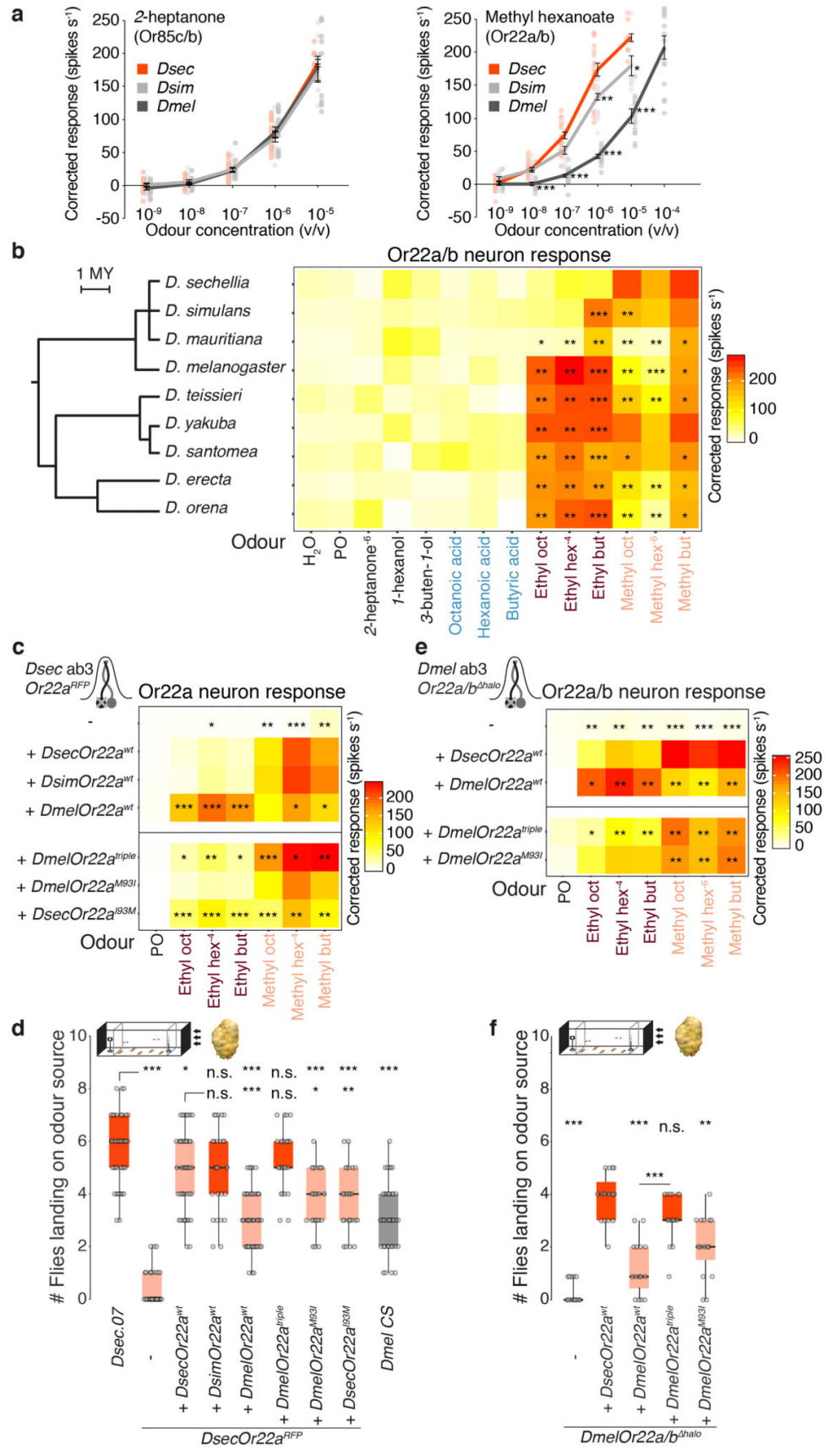

Fig. 3. Tuning of OR22a is important for noni attraction.

a, Dose-dependent responses of Or85c/b (left) and Or22a/(b) neurons (right) in the indicated species to 2-heptanone and methyl hexanoate, respectively (mean \pm SEM and individual data points, $\mathrm{n}=7-20$, females). Significant differences to $D$. sechellia responses are shown (a, $\mathbf{b}$, c and e: pairwise Wilcoxon rank-sum test, $P$ values adjusted for multiple comparisons using the B\&H method): *** $P<0.001 ; * * P<0.01 ; * P<0.05$. 
b, Responses of Or22a/(b) neurons to noni odours across the D. melanogaster species subgroup; $\mathrm{MY}=$ million years $(\mathrm{n}=5-20$, females). D. sechellia responses as in Fig. 2a. Significant differences to $D$. sechellia ester responses are shown.

c, Responses of $D$. sechellia Or22a neurons expressing wild-type (top) or mutant (bottom) versions of $\operatorname{Or} 22 a$ at the Or22a locus ( $\mathrm{n}=10-11$, females). Significant differences to DsecOR22a ${ }^{\text {wt }}$ responses are shown (c and $\left.\mathbf{e}\right)$. Responses to methyl hexanoate at $10^{-4}$ here compared to $10^{-6}$ in $\mathbf{b}$ and $\mathbf{e}$.

d, Behavioural responses to noni fruit in the wind tunnel assay ( $\mathrm{n}=25-45$ experiments). Comparisons to Dsec.07 (top) and DsecOR22a ${ }^{\text {wt }}$ (below) responses are shown (d and f , Kruskal-Wallis test, Dunn's post-hoc correction). Salmon $=D$. sechellia genotypes with significantly different response to Dsec. 07 ; *** $P<0.001$; ** $P<0.01$; $* P<0.05$.

e, Responses of $D$. melanogaster Or $22 a / b$ mutant neurons expressing wild-type (top) or mutant (bottom) versions of Or22a ( $\mathrm{n}=5-7$, females). Boxplot representations of $\mathbf{b}, \mathbf{c}$ and $\mathbf{e}$ are shown in Extended Data Fig. 8g, h and i, respectively.

f, Behavioural responses to noni fruit in the wind tunnel assay ( $\mathrm{n}=20$ experiments). Comparisons to DsecOr22a ${ }^{w t}$ (top) and DmelOR22a ${ }^{\text {wt }}$ (below) responses are shown. Salmon $=$ genotypes with significantly different responses. 

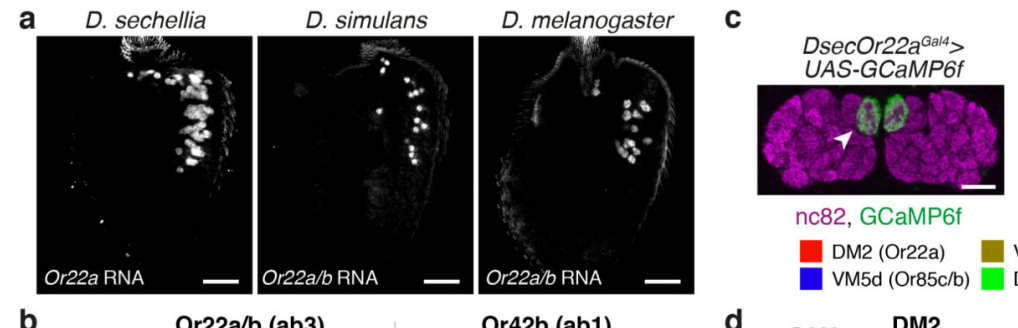

nc82, GCaMP6f

DM2 (Or22a)

d

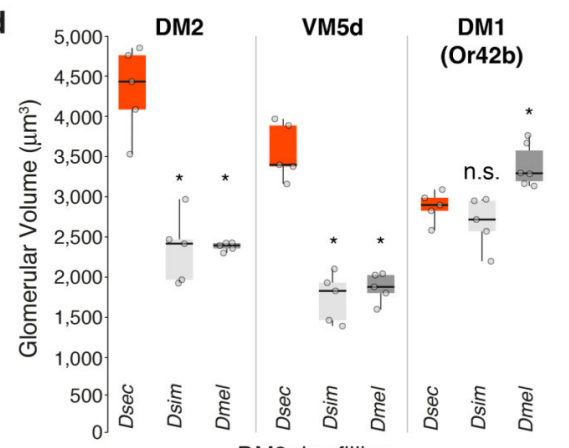

f

e
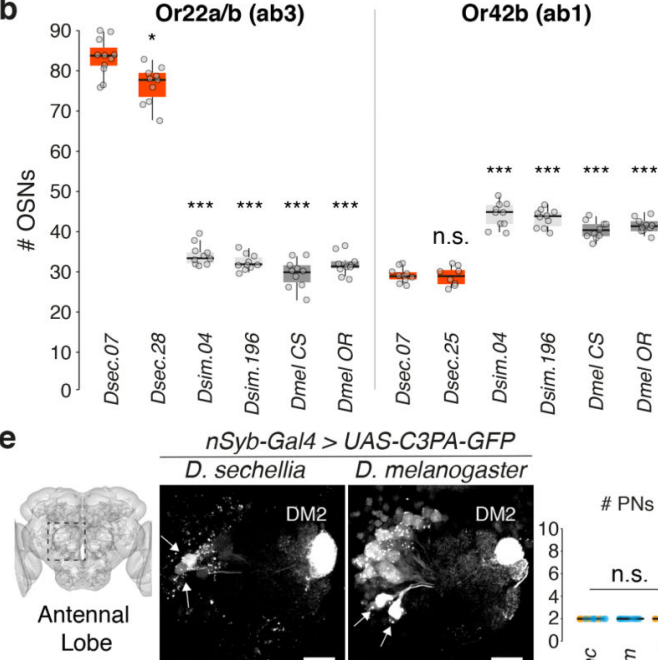

\begin{tabular}{c}
$n S y b-G a l 4>$ UAS-C3PA-GFP \\
\hline D. sechellia D. melanogaster
\end{tabular}
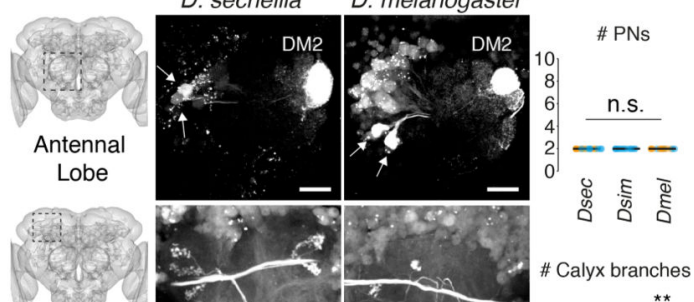

Mushroom Body Calyx

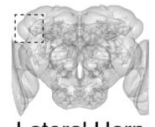

Lateral Horn

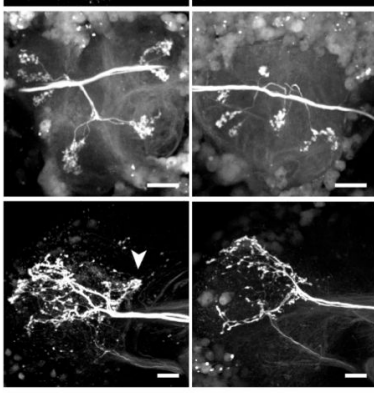

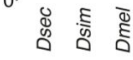

\# Calyx branches
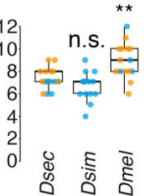

ळ

Dhotoactivation
Dye filling

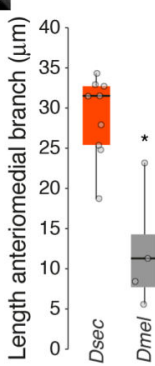

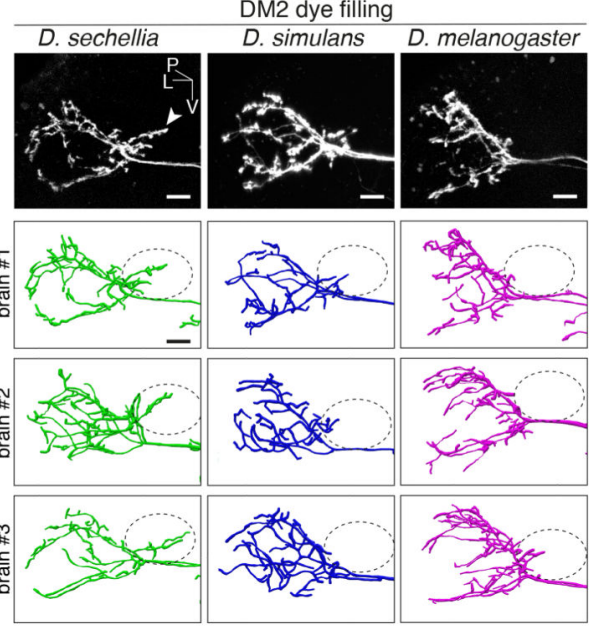

h

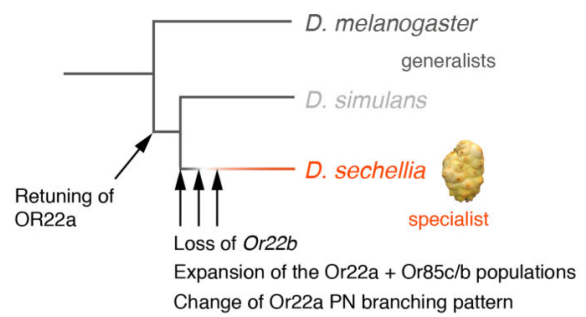

Fig. 4. Neuroanatomy of noni-sensing olfactory pathways.

a, Antennal Or22a/b RNA expression in different species. Scale bars, $25 \mu \mathrm{m}$.

b, Quantification of Or22a/(b) or Or42b OSNs ( $\mathrm{n}=8$-11, females). Comparisons to Dsec.07 are shown (pairwise Wilcoxon rank-sum test, $P$ values adjusted for multiple comparisons using the B\&H method, $\mathbf{b}, \mathbf{d}, \mathbf{e})$ : *** $P<0.001$; ** $P<0.01$; $P<0.05$; n.s. $P>0.05$.

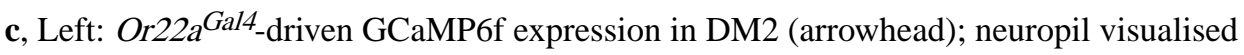
with nc82 (magenta). Scale bar, $25 \mu \mathrm{m}$. Right: Antennal lobe (AL) glomerular segmentation in $D$. sechellia (Extended Data Fig. 3). Scale bar, $50 \mu \mathrm{m}$. 
d, Quantification of DM2, VM5d and DM1 volumes ( $\mathrm{n}=5$ females).

e, DM2 projection neurons (PNs) labelled via photoactivation in D. sechellia (DsecnSybGal4/UAS-C3PA-GFP) and D. melanogaster (UAS-SPA-GFP/UAS-C3PA-GFP;nSyb-Gal4/ $U A S-C 3 P A-G F P)$. Left: Image acquisition site. Top: AL with labelled PNs (arrows) and DM2 glomerulus; scale bar, $20 \mu \mathrm{m}$. Middle and bottom: PN innervation of mushroom body calyx and lateral horn (LH); scale bars, $10 \mu \mathrm{m}$. Arrowhead (e, f, g): extra anterio-medial branch in D. sechellia. Right: quantification of DM2 PNs (top) and calyx branches (bottom) ( $\mathrm{n}=14-17$ females).

f, LH arbours of dye-filled DM2 PNs. Genotypes: D. sechellia, D. melanogaster as in e, $D$. simulans: DsimOr22aGFP. Below: representative LH DM2 arbour traces. Ovals: location of D. sechellia-specific branch. P, posterior; L, lateral; V, ventral. Scale bars, $10 \mu \mathrm{m}$.

g, Left: single dye-filled DM2 PN in D. sechellia; scale bar, $50 \mu \mathrm{m}$. Middle: representative LH arbour traces of DM2 PNs in D. sechellia and D. melanogaster, scale bar, $10 \mu \mathrm{m}$. Right: quantification of anteriomedial branch length $(n=4-9$ females) (pairwise Wilcoxon ranksum test): $* P<0.05$.

$\mathbf{h}$, Evolution of structural and physiological changes in the Or22a pathway. 